\title{
A well-balanced positivity-preserving central-upwind scheme for shallow water equations on unstructured quadrilateral grids
}

\author{
Hamidreza Shirkhani, PhD Candidate, University of Ottawa, h.r.shirkhani@uottawa.ca
}

Abdolmajid Mohammadian, Associate Professor, University of Ottawa,majid.mohammadian@uottawa.ca

Ousmane Seidou, Associate Professor, University of Ottawa, oseidou@ uottawa.ca

Alexander Kurganov, Professor, Mathematics Department, Tulane University, kurganov@tulane.edua

\begin{abstract}
We introduce a new second-order central-upwind scheme for shallow water equations on the

unstructured quadrilateral grids. We propose a new technique for bottom topography approximation over quadrilateral cells as well as an efficient water surface correction procedure which guarantee the positivity of the computed fluid depth. We also design a new quadrature for the discretization of the source term, using which the new scheme exactly preserves "lake at rest" steady states. We demonstrate these features of the new scheme as well as its high resolution and robustness and its potential advantages over the triangular central-upwind scheme in a number of numerical examples.
\end{abstract}

Keywords: Shallow water equations, central-upwind scheme, unstructured quadrilateral grids

\section{Introduction}

In this paper we consider the two-dimensional (2D) shallow water equations (SWEs):

$$
\left\{\begin{array}{c}
h_{t}+(h u)_{x}+(h v)_{y}=0 \\
(h u)_{t}+\left(h u^{2}+\frac{1}{2} g h^{2}\right)_{x}+(h u v)_{y}=-g h B_{x} \\
(h v)_{t}+(h u v)_{x}+\left(h v^{2}+\frac{1}{2} g h^{2}\right)_{y}=-g h B_{y} .
\end{array}\right.
$$


Here, $h(x, y, t)$ is the water depth, $u(x, y, t)$ and $v(x, y, t)$ are the $x$ - and $y$ - velocities, respectively, $B(x, y)$ is bottom topography and $g$ is the gravitational constant. 2D SWEs are commonly used to simulate a wide range of problems in water resources engineering, modelling oceans, rivers and coastal areas, etc.

The system (1) admits several steady-state solutions. One of the practically most important steady states is a so-called "lake at rest" state satisfying,

$u \equiv v \equiv 0, h+B=$ const.

A good numerical method for the SWEs (1) should be well-balanced, that is, it should be capable to exactly preserve the "lake at rest" steady states (2). It should also preserve positivity of the water depth $h$.

Many numerical methods for SWEs were developed in past decades. We refer the reader, for example, to finite difference [1-4], finite element [4-9] and finite volume [4,10-15] methods. In this paper we focus on the finite volume method which are based on the integral form of (1) and thus are naturally designed to conserve the mass.

Central upwind scheme is one of the finite volume methods that is both well-balanced and positivity preserving. Central-upwind schemes are Riemann-problem-solver-free Godunov-type methods that were originally introduced in [16] for the general multidimensional systems of hyperbolic conservation law and further developed in [17-20]. In [21-22], the central- upwind scheme for the SWEs were developed in the one-dimensional (1D) and 2D cases using Cartesian grids. In [23], the central-upwind schemes were extended to unstructured triangular meshes, and in [24], they were also generalized for polygon cell-vertex meshes. 
The main goal of this paper is to develop a second-order well-balanced positivity preserving central-upwind scheme for (1) on unstructured quadrilateral grids. Such grids have been widely used in finite volume methods for various applications, in particular, for numerically solving incompressible Navier-Stokes, diffusion equations, semilinear elliptic and elliptic systems, see, e.g., [25-28] and references therein. In particular, quadrilateral grids have been used to develop finite volume methods for the 2D SWEs, see, e.g., [29-34]. Unstructured quadrilateral grids are popular since they allow one to relatively easily implement the local and adaptive mesh refinement techniques [35-36], increase the formal order of spatial accuracy of the scheme, and discretize the second- and higher-order terms [30,37]. Comparing to the triangular grids, one of the main advantages of the quadrilateral ones is that quadrilateral cells have more neighbouring cells and thus the quadrilateral time evolution procedure is typically more accurate.

The proposed quadrilateral central-upwind scheme is an extension of the triangular central upwind scheme from [23]. However, some of the ingredients of the triangular scheme cannot be directly carried to the quadrilateral case. For example, one cannot obtain a continuous piecewise linear approximation of the bottom topography. Instead, we introduce a new bottom topography approximation: In each quadrilateral cell the bottom topography function $B$ is replaced with four continuous linear pieces, each of which connects the values of $B$ at two of the neighbouring cell vertices with the approximation value of $B$ at the geometric center of the cell. Another novelty of our quadrilateral scheme is a new water surface reconstruction correction technique, required to guarantee the positivity of the water depth at the reconstruction step of the central-upwind scheme. To this end, we first perform a piecewise 
linear reconstruction of the water surface and then, in the cells where some values of the reconstructions fall below the corresponding values of the bottom topography, we replace the linear piece with four continuously matched linear pieces whose shape is similar to the bottom topography approximant in this cell. As we prove in Theorem 1, this guarantees the positivity of the water depth $h$. To ensure the well-balanced property of the proposed scheme, we design a special quadrature for the cell average of the geometric source term, which leads to a perfect balance of the source and fluxes for the "lake at rest " state.

To the best of our knowledge, the designed central-upwind scheme is among the first wellbalanced positivity preserving schemes on unstructured quadrilateral grids.

The paper is organized as follows. The proposed central-upwind scheme is described in Section 2 and its well-balanced and positivity preserving properties are proved in Sections 3 and 4 . In Section 5 , the new scheme is tested on a number of numerical experiments which demonstrate high accuracy robustness of the proposed scheme and also emphasize its potential advantages over its triangular counterpart. Finally, we finish the paper with concluding remarks in Section 6.

\section{Central-Upwind Scheme on Unstructured Quadrilateral Grids}

First, we introduce the water surface variable $w=h+B$ and rewrite the system (1) in the following equivalent form:

$U_{t}+F(U, B)_{x}+G(U, B)_{y}=S(U, B)$,

where

$U=(w, h u, h v)^{T}$, 
$F(U, B)=\left(h u, \frac{(h u)^{2}}{w-B}+\frac{g}{2}(w-B)^{2}, \frac{(h u)(h v)}{w-B}\right)^{T}$,

$G(U, B)=\left(h v, \frac{(h u)(h v)}{w-B}, \frac{(h u)^{2}}{w-B}+\frac{g}{2}(w-B)^{2}\right)^{T}$,

$S(U, B)=\left(0,-g(w-B) B_{x},-g(w-B) B_{y}\right)^{T}$.

Let the computational domain discretization $\Omega=\bigcup_{j=1}^{N} E_{j}$ be covered by a quadrilateral grids with the cells $E_{j}$ of size $\left|E_{j}\right|$. A typical cell $E_{j}$ together with its neighbours $E_{j k}, k=1,2,3,4$ are outlined in Fig. 1.

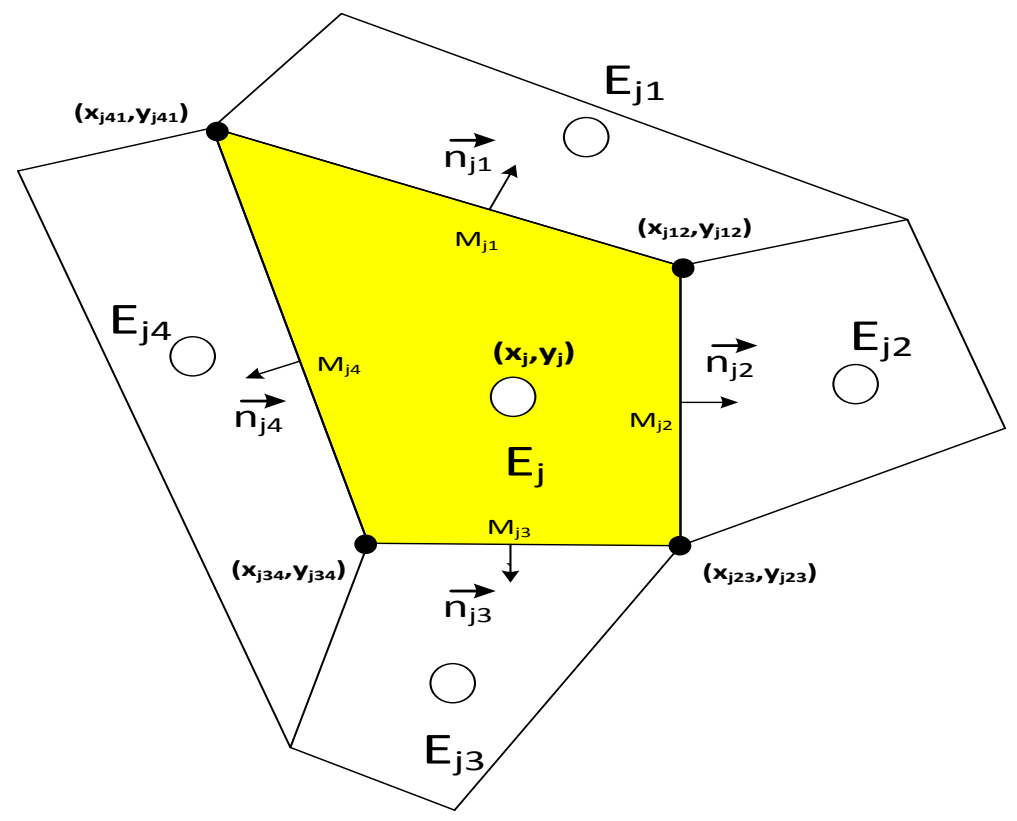

Fig. 1- An unstructured quadrilateral cell with its four neighbouring cells.

We denote by $\vec{n}_{j k}:=\left(\cos \left(\theta_{j k}\right), \sin \left(\theta_{j k}\right)\right)$ the outer unit normals of the corresponding sides of $E_{j}$ of length $l_{j k}, k=1,2,3,4$. The coordinates of the geometric center (center of mass) of the $E_{j}$ 
are denoted by $\left(x_{j}, y_{j}\right)$ and $M_{j k}:=\left(x_{j k}, y_{j k}\right), k=1,2,3,4$ is the midpoint of the $k$-th side of the quadrilateral $E_{j}$.

In the semi-discrete central-upwind scheme, the cell average of the computed solutions, $\bar{U}_{j}^{(t)} \approx \frac{1}{E_{j}} \int_{E_{j}} U(x, y, t) d x d y$, are evolved in time by solving the following system of ODEs:

$$
\begin{aligned}
\frac{d \bar{U}_{j}}{d t}= & -\frac{1}{\left|E_{j}\right|} \sum_{k=1}^{4} \frac{l_{j k} \cos \left(\theta_{j k}\right)}{a_{j k}^{i n}+a_{j k}^{\text {out }}}\left[a_{j k}^{i n} F\left(U_{j k}\left(M_{j k}\right), B\left(M_{j k}\right)\right)+a_{j k}^{\text {out }} F\left(U_{j}\left(M_{j k}\right), B\left(M_{j k}\right)\right)\right] \\
& -\frac{1}{\left|E_{j}\right|} \sum_{k=1}^{4} \frac{l_{j k} \sin \left(\theta_{j k}\right)}{a_{j k}^{\text {in }}+a_{j k}^{\text {out }}}\left[a_{j k}^{\text {in }} G\left(U_{j k}\left(M_{j k}\right), B\left(M_{j k}\right)\right)+a_{j k}^{\text {out }} G\left(U_{j}\left(M_{j k}\right), B\left(M_{j k}\right)\right)\right] \\
& +\frac{1}{\left|E_{j}\right|} \sum_{k=1}^{4} l_{j k} \frac{a_{j k}^{\text {in }} a_{j k}^{\text {out }}}{a_{j k}^{\text {in }}+a_{j k}^{\text {out }}}\left[U_{j k}\left(M_{j k}\right)-U_{j}\left(M_{j k}\right)\right]+\bar{S}_{j},
\end{aligned}
$$

which can be derived similarly to the derivation procedure proposed for a triangular grids in $[20,23]$. Notice that all the indexed quantities in $(8)$ are functions of $t$, but from now on we omit this dependence for the sake of brevity.

The values $U_{j}\left(M_{j k}\right)$ and $U_{j k}\left(M_{j k}\right)$ are the values at $M_{j k}$ of the two polynomial pieces reconstructed in cells $E_{j}$ and $E_{j k}$, respectively. The corresponding piecewise linear reconstruction is:

$\widetilde{U}_{j}(x, y)=\bar{U}_{j}+\left(U_{x}\right)_{j}\left(x-x_{j}\right)+\left(U_{y}\right)_{j}\left(y-y_{j}\right)$

To minimize the oscillations, the slopes $\left(U_{x}\right)_{j}$ and $\left(U_{y}\right)_{j}$ are to be computed using a nonlinear limiter. We propose the following minmod-type limiter which will be applied in a component wise manner. Consider the $i$ th component of $U$, we first construct four linear interpolations $L_{j}^{12}, L_{j}^{23}, L_{j}^{34}$ and $L_{j}^{41}$, each of which is obtained by considering the three points at the geometric center of $E_{j}$ and corresponding two neighbouring cells. For example, $L_{j}^{12}$ is obtained by passing the plane through $\left(x_{j}, y_{j}, \bar{U}_{j}^{(i)}\right),\left(x_{j 1}, y_{j 1}, \bar{U}_{j 1}^{(i)}\right)$ and $\left(x_{j 2}, y_{j 2}, \bar{U}_{j 2}^{(i)}\right)$. Notice that all of the four 
obtained interpolants are conservative in the cell $E_{j}$ by construction. We then select the linear piece with the smallest magnitude of the gradient, say $L_{j}^{k m}$, and we set:

$\left(\left(U_{x}\right)_{j}^{(i)},\left(U_{y}\right)_{j}^{(i)}\right)=\nabla L_{j}^{k m}$

In order to further minimize the reconstruction oscillations, the reconstructed values calculated at the points $M_{j k}, k=1,2,3,4$ are checked. If the reconstructed value of $U_{j}^{(i)}\left(M_{j k}\right)$ is not between the cell averages $\bar{U}_{j}^{(i)}$ and $\bar{U}_{j k}^{(i)}$, we set

$\left(\left(U_{x}\right)_{j}^{(i)},\left(U_{y}\right)_{j}^{(i)}\right)=0$.

The quantity $\overline{S_{j}}$ which is used in (8) is a discretization of the cell averages of the source term:

$\bar{S}_{j}(t) \approx \frac{1}{\left|E_{j}\right|} \int_{E_{j}} S(U(x, y, t), B(x, y)) d x d y$.

The well-balanced discretization form of the source term $\bar{S}_{j}$ will be discussed in Section 3 .

Finally $a_{j k}^{i n}$ and $a_{j k}^{\text {out }}$ in (8) are the directional local speeds of propagation at the $k$ th interface of the cell $E_{j}$, which are defined as the smallest and largest eigenvalues of the Jacobian

$V_{j k}=\cos \left(\theta_{j k}\right) \frac{\partial F}{\partial U}+\sin \left(\theta_{j k}\right) \frac{\partial G}{\partial U}$

with

$$
\begin{aligned}
& a_{j k}^{\text {in }}=-\min \left\{\lambda_{1}\left[V_{j k}\left(U_{j}\left(M_{j k}\right)\right)\right], \lambda_{1}\left[V_{j k}\left(U_{j k}\left(M_{j k}\right)\right)\right], 0\right\}, \\
& a_{j k}^{\text {out }}=\max \left\{\lambda_{3}\left[V_{j k}\left(U_{j}\left(M_{j k}\right)\right)\right], \lambda_{3}\left[V_{j k}\left(U_{j k}\left(M_{j k}\right)\right)\right], 0\right\},
\end{aligned}
$$

where $\lambda_{1}\left[V_{j k}\right] \leq \lambda_{2}\left[V_{j k}\right] \leq \lambda_{3}\left[V_{j k}\right]$ are the eigenvalues of $V_{j k}$.

It should be noted that if the $a_{j k}^{i n}$ and $a_{j k}^{o u t}$ are zero or very close to zero, the semi discrete scheme (8) will reduce to: 


$$
\begin{aligned}
& \frac{d \bar{U}_{j}}{d t}=-\frac{1}{\left|E_{j}\right|} \sum_{k=1}^{4} \frac{l_{j k} \cos \left(\theta_{j k}\right)}{2}\left[a_{j k}^{i n} F\left(U_{j k}\left(M_{j k}\right), B\left(M_{j k}\right)\right)+a_{j k}^{o u t} F\left(U_{j}\left(M_{j k}\right), B\left(M_{j k}\right)\right)\right]- \\
& \frac{1}{\left|E_{j}\right|} \sum_{k=1}^{4} \frac{l_{j k} \sin \left(\theta_{j k}\right)}{2}\left[a_{j k}^{i n} G\left(U_{j k}\left(M_{j k}\right), B\left(M_{j k}\right)\right)+a_{j k}^{o u t} G\left(U_{j}\left(M_{j k}\right), B\left(M_{j k}\right)\right)\right]+\bar{S}_{j} .
\end{aligned}
$$

In all the numerical experiments reported in this paper, we have used (15) instead of (8) whenever $a_{j k}^{i n}+a_{j k}^{\text {out }}<10^{-8}$.

The semi-discretization (8) is a system of ODEs, which has to be integrated in time using a sufficiently accurate and stable ODE solver. In all of the numerical experiments, we have used the third-order strong stability preserving (SSP) Runge-Kutta method [38,39].

\subsection{Piecewise linear approximation of the bottom}

In this section, we describe how the bottom topography function $B(x, y)$ is replaced by its continuous piecewise linear approximation $\tilde{B}(x, y)$. To this end, we first define values of the bottom topography $B_{j_{\kappa}}, \kappa=1,2 ; 2,3 ; 3,4 ; 4,1$ at the vertices of the cell $E_{j}$. If the function $B(x, y)$ is continuous at the vertices we simply take $B_{j_{\kappa}}=B\left(x_{j_{\kappa}}, x_{j_{\kappa}}\right)$, otherwise we use the following formula:

$B_{j_{\kappa}}=\frac{1}{2}\left(\max _{\zeta^{2}+\eta^{2}=1} \lim _{h, l \rightarrow 0} B\left(\tilde{x}_{j k}+h \zeta, \tilde{y}_{j k}+l \eta\right)+\min _{\zeta^{2}+\eta^{2}=1} \lim _{h, l \rightarrow 0} B\left(\tilde{x}_{j k}+h \zeta, \tilde{y}_{j k}+\right.\right.$ $l \eta))$

By $B_{j k}$ we denote the value of the bottom topography at the center of the $k$ th side of the cell $E_{j}$, which equals to the average of the values of the bottom topography at the two vertices of the corresponding side, for example, $B_{j 1}=\frac{B_{j_{1,2}}+B_{j_{4,1}}}{2}$. Then, we approximate the value of the bottom topography at the geometric center of the cell $E_{j}$ as follows:

$\int_{E_{j}} B(x, y) d x d y \approx \sum_{k=1}^{4} \mu_{k} B_{j k}=: B_{j}$ 
where $\mu_{k}=\left|A_{j k}\right| /\left|E_{j}\right|$ and $\left|A_{j k}\right|$ is the area of the triangle whose first vertex is $\left(x_{j}, y_{j}\right)$ and two other vertices are the vertices of the $k$ th side of the cell $E_{j}$, see Fig. 2 .

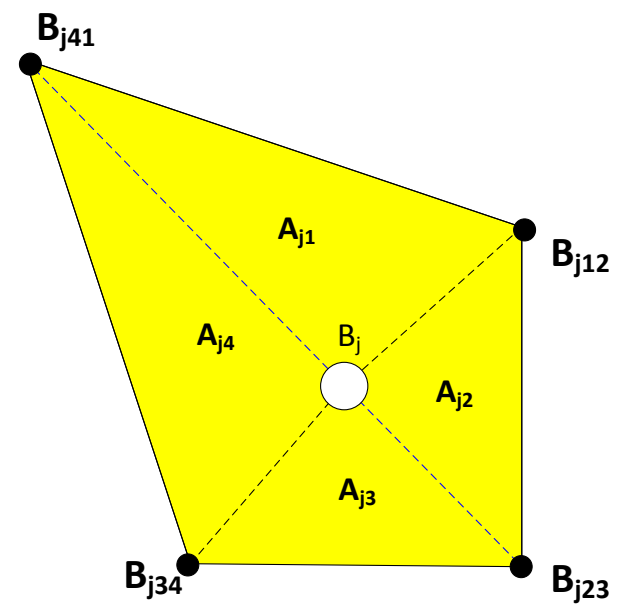

Fig. 2 - Subdividing cell $E_{j}$ into four triangles $A_{j 1}, A_{j 2}, A_{j 3}$ and $A_{j 4}$

This way, the bottom approximation in each cell will consist of four linear pieces that continuously match along the segments $\left[B_{j}, B_{j_{\kappa}}\right], \kappa=1,2 ; 2,3 ; 3,4 ; 4,1$.

Finally, we obtain the continuous piecewise linear approximation of the bottom topography $\tilde{B}(x, y) \approx B(x, y)$.

\subsection{Positivity Preserving Reconstruction for Water Surface Elevation}

In this section, we propose a simple and efficient algorithm for the positivity preserving reconstruction of the water surface level. Generally, the reconstruction of $w$ is positivity preserving if it leads to nonnegative computed values of water depth $h_{j}\left(M_{j k}\right), k=1,2,3,4$, for all the cells $E_{j}$ in the computational domain. First, we compute $h_{j}\left(M_{j k}\right)$ using the reconstructed values of $\widetilde{w}$ and $\widetilde{B}$ as follows:

$h_{j}\left(M_{j k}\right)=\widetilde{w}_{j}\left(M_{j k}\right)-B_{j k}, k=1,2,3,4$. 
However, some of the obtained values of $h_{j}\left(M_{j k}\right)$ may be negative even where $\bar{w}_{j} \geq B_{j}$. Therefore, we may need to correct the original reconstruction for $w$ to ensure $h_{j}\left(M_{j k}\right) \geq 0$ for all $j, k$. In the proposed correction procedure, the correction is required when we have $\widetilde{w}\left(x_{j_{\kappa}}, y_{j_{\kappa}}\right)<B_{j_{\kappa}}$ at any of the vertices of the cell $E_{j}$ (note that since $\bar{w}_{j} \geq B_{j}$ it is impossible to have $\widetilde{w}\left(x_{j_{\kappa}}, y_{j_{\kappa}}\right)<B_{j_{\kappa}}$ at all the four vertices of the cell $\left.E_{j}\right)$. In this case we replace the original reconstruction of $w$ by four linear planes over the same four triangles, as used in Section 2.1, to obtain the bottom topography approximation. To this end, we need to specify the new (corrected) values at each of the vertices. we set $w_{j_{\kappa}}^{c o r r}=B_{j_{\kappa}}$ at the vertices at which $\widetilde{w}\left(x_{j_{\kappa}}, y_{j_{\kappa}}\right)<B_{j_{\kappa}}$ and for the rest of the vertices we set $w_{j_{\kappa}}^{\text {corr }}=h_{j}^{*}+B_{j_{\kappa}}$ where, $h_{j}^{*}=\frac{\bar{w}_{j}-B_{j}}{\sum_{1}^{4} \mu_{k} \alpha_{k}}, \alpha=\left\{\begin{array}{lr}1, & \text { if } \widetilde{w} \geq \widetilde{B} \text { at both ends of the } k^{\text {th }} \text { side of } E_{j}, \\ \frac{1}{2}, & \text { if } \widetilde{w} \geq \widetilde{B} \text { at one of the ends of the } k^{\text {th }} \text { side of } E_{j}, \\ 0, & \text { if } \widetilde{w}<\widetilde{B} \text { at both ends of the } k^{\text {th }} \text { side of } E_{j},\end{array}\right.$

and, as before, $\mu_{k}=\left|A_{j k}\right| /\left|E_{j}\right|$. We also set $w_{j}^{\text {corr }}=\bar{w}_{j}$ to be the value of $w$ at the geometric center of $E_{j}$. One can show that the corrected reconstruction of $w$, which consists of four linear pieces in the cell $E_{j}$, is conservative. Indeed,

$$
\begin{aligned}
& \frac{1}{\left|E_{j}\right|} \int_{E_{j}}\left[\widetilde{w}_{j}^{\text {corr }}(x, y)-B(x, y)\right] d x d y=\frac{1}{\left|E_{j}\right|} \sum_{k=1}^{4} \int_{A_{j k}}\left[\widetilde{w}_{j}^{c o r r}(x, y)-B(x, y)\right] d x d y \\
& =\sum_{k=1}^{4} \frac{\left(\bar{w}_{j}-B_{j}\right)+2 \alpha_{j k} h^{*}}{3} \mu_{j k}=\frac{\bar{w}_{j}-B_{j}}{3}+\frac{2}{3} h^{*} \sum_{k=1}^{4} \alpha_{j k} \mu_{j k}=\bar{w}_{j}-B_{j},
\end{aligned}
$$

which implies that

$$
\frac{1}{\left|E_{j}\right|} \int_{E_{j}} \widetilde{w}_{j}^{c o r r}(x, y) d x d y=\bar{w}_{j} .
$$


Also note that since the linear pieces of the corrected reconstruction of $w$ are over the same triangles $A_{j k}, k=1,2,3,4$ as the linear pieces of the $\tilde{B}$, all of the obtained values of $h$ will be nonnegative.

Remark. We note that similarly to the correction procedures introduced in [22] and [23], the correction procedure proposed here locally reduces the order of accuracy of the developed central-upwind scheme to the first-order. However, this occurs only in the cells, which are almost dry and thus the global accuracy of the computed results is not expected to be heavily affected by the local order reduction unless the entire computational domain contains mostly (almost) dry cells.

Having the corrected reconstruction $\widetilde{w}$, we continue with the computation of the velocities $u$ and $v$, and the one-sided local speeds needed in (8). Since the computed values of water depth $h$ may be very small (or even zero), we use the following desingularization procedure to calculate the velocities (see [23]):

$u=\frac{\sqrt{2} h(h u)}{\sqrt{h^{4}+\max \left(h^{4}, \varepsilon\right)}}$ and $v=\frac{\sqrt{2} h(h v)}{\sqrt{h^{4}+\max \left(h^{4}, \varepsilon\right)}}$

where $\varepsilon$ is a prescribed positive tolerance. In all our numerical tests, we have used $\varepsilon=$ $\max \left\{|E j|^{2}\right\}$. After computing $h, u$, and $v$, we recompute the flux vectors $F$ and $G$ as

$$
\begin{aligned}
& (h u)=h \cdot u, \\
& (h v)=h \cdot v, \\
& F(U, B)=\left(h u, h u \cdot u+\frac{g}{2}(w-B)^{2}, h u \cdot v\right)^{T}, \\
& G(U, B)=\left(h v, h v \cdot u, h v \cdot v+\frac{g}{2}(w-B)^{2}\right)^{T},
\end{aligned}
$$


at the corresponding points used in (8). Now, we are also able to compute the one-sided speeds of propagation:

$a_{j k}^{i n}=-\min \left\{u_{j}^{\theta}\left(M_{j k}\right)-\sqrt{g h_{j}\left(M_{j k}\right)}, u_{j k}^{\theta}\left(M_{j k}\right)-\sqrt{g h_{j k}\left(M_{j k}\right)}, 0\right\}$,

$a_{j k}^{\text {out }}=\max \left\{u_{j}^{\theta}\left(M_{j k}\right)+\sqrt{g h_{j}\left(M_{j k}\right)}, u_{j k}^{\theta}\left(M_{j k}\right)+\sqrt{g h_{j k}\left(M_{j k}\right)}, 0\right\}$,

$u_{j}^{\theta}\left(M_{j k}\right)=\cos \left(\theta_{j k}\right) u_{j}\left(M_{j k}\right)+\sin \left(\theta_{j k}\right) v_{j}\left(M_{j k}\right), u_{j k}^{\theta}\left(M_{j k}\right)=\cos \left(\theta_{j k}\right) u_{j k}\left(M_{j k}\right)+\sin \left(\theta_{j k}\right) v_{k j}\left(M_{j k}\right)$

where $u_{j}^{\theta}\left(M_{j k}\right)$ and $u_{j k}^{\theta}\left(M_{j k}\right)$ are the normal velocities at the midpoint of the $k$ th side $M_{j k}$.

\section{Well-Balanced Discretization of the Source Term}

Here, we propose a new well-balanced discretization of the source term for unstructured quadrilateral grids. Indeed, the proposed semi-discrete central-upwind scheme (8) includes the cell averages of the source term $\bar{S}_{j}$. Recall that a well- balanced scheme should exactly preserve steady-state solutions. For this aim, a specific quadrature must be designed in order to exactly preserve a "lake at rest" with $w \equiv C, u \equiv v \equiv 0$, where $C$ is a constant. In this case, the two momentum equations in (8) will become:

$$
\begin{aligned}
& -\frac{g}{2\left|E_{j}\right|} \sum_{k=1}^{4} l_{j k} \cos \left(\theta_{j k}\right)\left(C-B_{j k}\right)^{2}+\bar{S}_{j}^{(2)}=0, \\
& -\frac{g}{2\left|E_{j}\right|} \sum_{k=1}^{4} l_{j k} \sin \left(\theta_{j k}\right)\left(C-B_{j k}\right)^{2}+\bar{S}_{j}^{(3)}=0
\end{aligned}
$$

We now design an appropriate quadrature in order to satisfy the well-balancing conditions (23).

First, we use the divergence theorem, $\int_{E_{j}} \operatorname{div} \vec{\varsigma} d x d y=\int_{\partial E_{j}} \vec{\varsigma} \cdot \vec{n} d s$, to obtain

$$
\begin{aligned}
& \bar{S}_{j}^{(2)}=-\frac{g}{\left|E_{j}\right|} \int_{E_{j}}(w(x, y)-B(x, y)) B_{x} d x d y \\
& =-\frac{g}{\left|E_{j}\right|} \sum_{k=1}^{4} \int_{\partial E_{j k}} \frac{(w(x, y)-B(x, y))^{2}}{2} \cos \left(\theta_{j k}\right) d s-\frac{g}{\left|E_{j}\right|} \int_{E_{j}}(w(x, y)-B(x, y)) w_{x} d x d y,
\end{aligned}
$$


where $\partial E_{j k}$ is the $k$ th side of the quadrilateral $E_{j}, k=1,2,3,4$. Due to the correction of water surface reconstruction, it may happen that we have four linear planes, instead of one, for each cell $E_{j}$, with different $w_{x}$. Thus, the second integral on the RHS of (24) should be subdivided into four integrals over $A_{j k}, k=1,2,3,4$ (see Fig. 2):

$-\frac{g}{\left|E_{j}\right|} \int_{E_{j}}(w(x, y)-B(x, y)) w_{x} d x d y=-\frac{g}{\left|E_{j}\right|} \sum_{k=1}^{4} \int_{A_{j k}}(w(x, y)-B(x, y)) w_{x} d x d y$.

Finally, applying the midpoint rule to the integrals on the RHS of (24) and (25) and replacing $B$ with its piecewise linear approximant $\widetilde{B}$, we arrive at the following quadrature for the cell average $\bar{S}_{j}^{(2)}$,:

$\bar{S}_{j}^{(2)}=\frac{g}{\left|E_{j}\right|} \sum_{k=1}^{4}\left[\frac{\left(w\left(M_{j k}\right)-B\left(M_{j k}\right)\right)^{2}}{2} \cos \left(\theta_{j k}\right) l_{j k}-\left(\bar{w}_{j}-B_{j}\right)\left(w_{x}\right)_{j k}\right]$.

Similarly, we obtain the quadrature for the cell average $\bar{S}_{j}^{(3)}$,

$\bar{S}_{j}^{(3)}=\frac{g}{\left|E_{j}\right|} \sum_{k=1}^{4}\left[\frac{\left(w\left(M_{j k}\right)-B\left(M_{j k}\right)\right)^{2}}{2} \sin \left(\theta_{j k}\right) l_{j k}-\mu_{j k}\left(\bar{w}_{j}-B_{j}\right)\left(w_{y}\right)_{j k}\right]$.

It should be noted that for the lake at rest with $U \equiv(C, 0,0)^{T}$, we have $w \equiv C$ and $w_{y} \equiv w_{x} \equiv$ 0 , and the designed quadratures (26) and (27) satisfy (23).

\section{Positivity preserving property of the scheme}

In this section, we prove the positivity preserving property of the proposed scheme. The prove is valid either for the forward Euler or any higher-order SSP Runge-Kutta method used for time discretization of the system of ODEs. For the sake of brevity, we formulate the result for the forward Euler method. 
Theorem 1. Consider the proposed central-upwind scheme (8), (9), (22), (26) and (27) for the system (3)-(7). Assume that the forward Euler method is used for solving the system of ODEs (8) while $w_{j}^{n} \geq B_{j}$ for all jat time $t=t^{n}$. Then, $w_{j}^{n+1} \geq B_{j}$ for all $j$ at $t=t^{n+1}$, provided that $\Delta t \leq \frac{1}{2 a} \min _{j, k}\left\{d_{j k}\right\}$

where $a=\max _{j, k}\left\{a_{j k}^{\text {in }}, a_{j k}^{\text {out }}\right\}$ and $d_{j k}$ is the distance between the center of mass of cell $E_{j}$ and its $k$-th side.

Proof. Using the forward Euler time stepping method for the first component of the system (8) we obtain

$$
\begin{aligned}
\bar{w}_{j}^{n+1}=\bar{w}_{j}^{n}- & \frac{\Delta t}{\left|E_{j}\right|} \sum_{k=1}^{4} \frac{l_{j k} \cos \left(\theta_{j k}\right)}{a_{j k}^{\text {in }}+a_{j k}^{\text {out }}}\left[a_{j k}^{\text {in }}(h u)_{j k}\left(M_{j k}\right)+a_{j k}^{\text {out }}(h u)_{j}\left(M_{j k}\right)\right] \\
& -\frac{\Delta t}{\left|E_{j}\right|} \sum_{k=1}^{4} \frac{l_{j k} \sin \left(\theta_{j k}\right)}{a_{j k}^{\text {in }}+a_{j k}^{\text {out }}}\left[a_{j k}^{\text {in }}(h v)_{j k}\left(M_{j k}\right)+a_{j k}^{\text {out }}(h v)_{j}\left(M_{j k}\right)\right] \\
& +\frac{\Delta t}{\left|E_{j}\right|} \sum_{k=1}^{4} \frac{a_{j k}^{\text {in }} a_{j k}^{\text {out }}}{a_{j k}^{\text {in }}+a_{j k}^{\text {out }}}\left[w_{j k}\left(M_{j k}\right)-w_{j}\left(M_{j k}\right)\right] .
\end{aligned}
$$

Since the piecewise linear interpolant $\widetilde{B}$ of the bottom topography is continuous, one has:

$w_{j k}\left(M_{j k}\right)-w_{j}\left(M_{j k}\right)=h_{j k}\left(M_{j k}\right)-h_{j}\left(M_{j k}\right)$.

In addition, (16), (17) and $\bar{w}_{j}^{n}=\sum_{k=1}^{4} \mu_{j k} w_{j}\left(M_{j k}\right)$ imply that

$\bar{w}_{j}^{n}-B_{j}=\sum_{k=1}^{4} \mu_{j k} h_{j}\left(M_{j k}\right)$

From (30) and (31), replacing $B_{j}$ from both sides of (31) and using the notation (22), we write,

$\bar{h}_{j}^{n+1}=\frac{\Delta t}{\left|E_{j}\right|} \sum_{k=1}^{4} h_{j k}\left(M_{j k}\right) \frac{l_{j k} a_{j k}^{i n}}{a_{j k}^{i n}+a_{j k}^{o u t}}\left[a_{j k}^{o u t}-u_{j k}^{\theta}\left(M_{j k}\right)\right]$ 


$$
+\sum_{k=1}^{4} h_{j k}\left(M_{j k}\right)\left(\mu_{j k}-\frac{\Delta t}{\left|E_{j}\right|} \frac{l_{j k} a_{j k}^{o u t}}{a_{j k}^{i n}+a_{j k}^{o u t}}\left[a_{j k}^{i n}+u_{j}^{\theta}\left(M_{j k}\right)\right]\right)
$$

From the definitions of the local speeds (22) we obtain that $a_{j k}^{o u t} \geq u_{j k}^{\theta}\left(M_{j k}\right)$ and therefore, all terms in the first sum on the RHS of (32) are nonnegative since the corrected reconstruction for w guarantees that $h_{j k}\left(M_{j k}\right) \geq 0$ for all $j$ and $k=1,2,3,4$. We also obtain that

$\frac{\Delta t}{\left|E_{j}\right|} \frac{l_{j k} a_{j k}^{o u t}}{a_{j k}^{i n}+a_{j k}^{\text {out }}}\left[a_{j k}^{\text {in }}+u_{j}^{\theta}\left(M_{j k}\right)\right] \leq \frac{\Delta t}{\left|E_{j}\right|} l_{j k} a_{j k}^{\text {out }}$

Thus, the positivity of the second termon the RHS of (33) is guaranteed provided

$\Delta t \leq \frac{\mu_{j k}\left|E_{j}\right|}{l_{j k} a_{j k}^{\text {out }}}$

Recall that $\mu_{k}=A_{j k} /\left|E_{j}\right|$ and $A_{j k}=\frac{d_{j k} l_{j k}}{2}$, hence (34) becomes $\Delta t \leq \frac{1}{2 a} \min _{j, k}\left\{d_{j k}\right\}$, and this completes the proof of the thoreme.

Remark. In all our numerical tests, adaptive time step is implemented in all the numerical examples and it is selected to satisfy the condition (28).

\section{Numerical Experiments}

In this section, we apply our proposed central-upwind scheme on unstructured quadrilateral grids to several test problems. We perform the numerical experiments using the organized unstructured grids shown in Fig. 3. However, we would like to point out that the proposed method can be applied on any quadrilateral grids. In all examples below, the gravitational constant is $g=1$. 


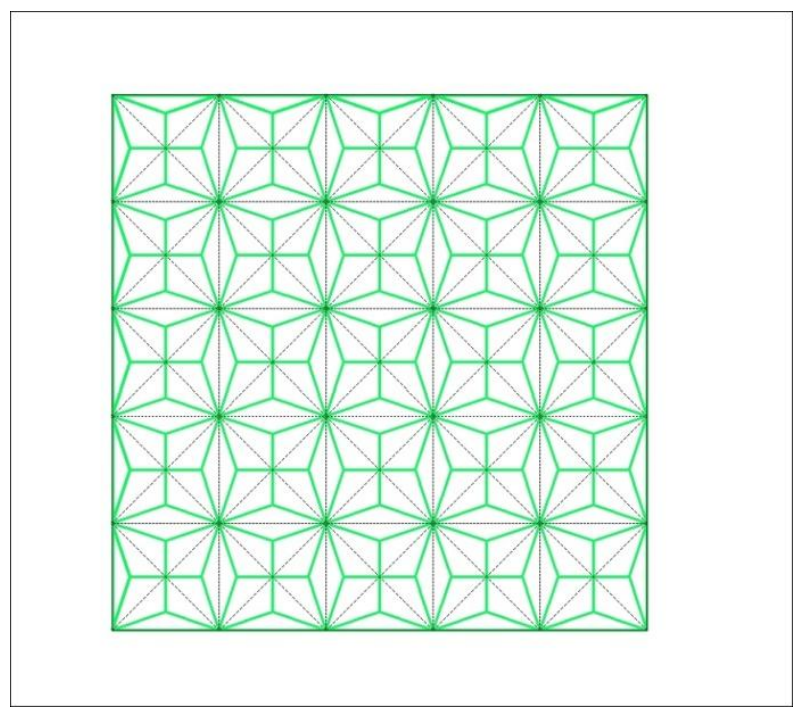

Fig. 3 - Organized unstructured quadrilateral mesh.

\section{Example 1 - Accuracy test}

In the first example, we experimentally test the order of accuracy of the proposed scheme. The scheme is applied to the following initial data and bottom topography:

$w(x, y, 0)=1, u(x, y, 0)=0.3, B(x, y)=0.5 \exp \left(-25(x-1)^{2}-50(y-0.5)^{2}\right)$.

The computational domain is $[0,2] \times[0,1]$ and the transparency conditions are imposed at all its boundaries. We use a fine grid with $6 \times 400 \times 400$ cells and consider the obtained results as a reference solution. The solution converges to the steady state by the time $t=0.07$. The $L^{1}$ and $L^{\infty}$-errors are presented in Table 1. 
Table 1 - Example1: $L^{1}$ - and $L^{\infty}$-errors and numerical orders of accuracy.

\begin{tabular}{|c|c|c|c|c|}
\hline Number of cells & $L^{1}$-error & order & $L^{\infty}$-error & order \\
\hline $6 \times 50 \times 50$ & $3.61 e-04$ & - & $7.45 e-03$ & - \\
\hline $6 \times 100 \times 100$ & $1.48 e-04$ & 1.29 & $3.09 e-03$ & 1.27 \\
\hline $6 \times 200 \times 200$ & $4.27 e-05$ & 1.80 & $9.38 e-04$ & 1.72 \\
\hline
\end{tabular}

Comparing the results with those reported in [23] shows that the obtained orders are higher.

The water surface computed at $t=0.07$ using the mesh with $6 \times 50 \times 50$ quadrilaterals is shown in Fig. 4. Although a relatively coarse mesh has been used, the water surface is well resolved by the scheme.
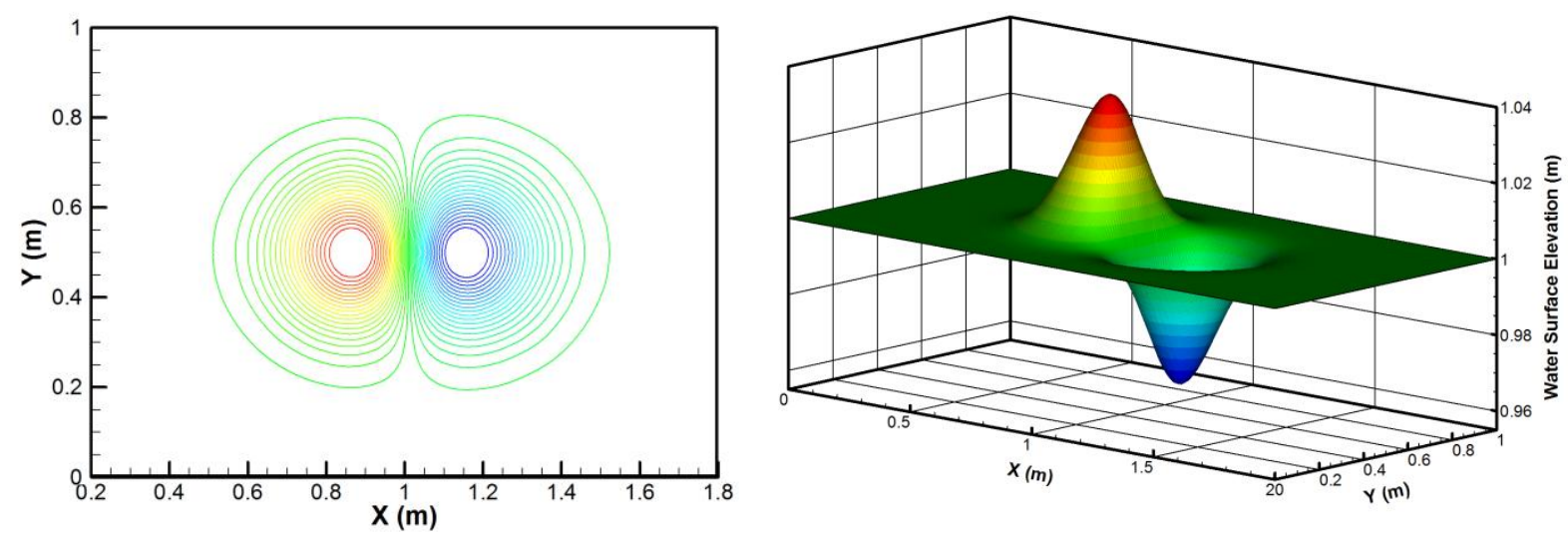

Fig. 4 - Example 1: Contour plot (left) and 3D view (right) of $w$.

\section{Example 2 - Small Perturbation over an Exponential Hump}

In this numerical experiment, originally proposed in [40] and then widely used in the literature $[2,15,21,23]$, we investigate the capability of the proposed scheme to accurately capture the propagation of a small perturbation of the "lake at rest" steady state. The computational domain is $[0,2] \times[0,1]$ and the bottom consists of an elliptically shaped hump: 
$B(x, y)=0.8 \exp \left(-5(x-0.9)^{2}-50(y-0.5)^{2}\right)$.

The water initially is at rest and its surface is flat everywhere except for $0.05<x<0.15$ :

$w(x, y, 0)=\left\{\begin{array}{rc}1+\epsilon, & 0.05<x<0.15, \quad u(x, y, 0) \equiv v(x, y, 0) \equiv 0, \\ 1, & \text { otherwise, }\end{array}\right.$

where $\varepsilon$ is the perturbation height. The transparency boundary conditions are implemented at vertical boundaries of the domain while the horizontal boundaries are periodic. First, in order to numerically verify the well-balanced property of the proposed scheme, a very small $\varepsilon=10^{-12}$ is selected and the solution is computed using a coarse mesh with $6 \times 10 \times$ 10cells. Max $(w-1)$ as a function of $t$ is plotted in Fig. 5 and it can be clearly seen that no instabilities are developed and the balance between the fluxes and the geometric source terms is exactly preserved numerically.

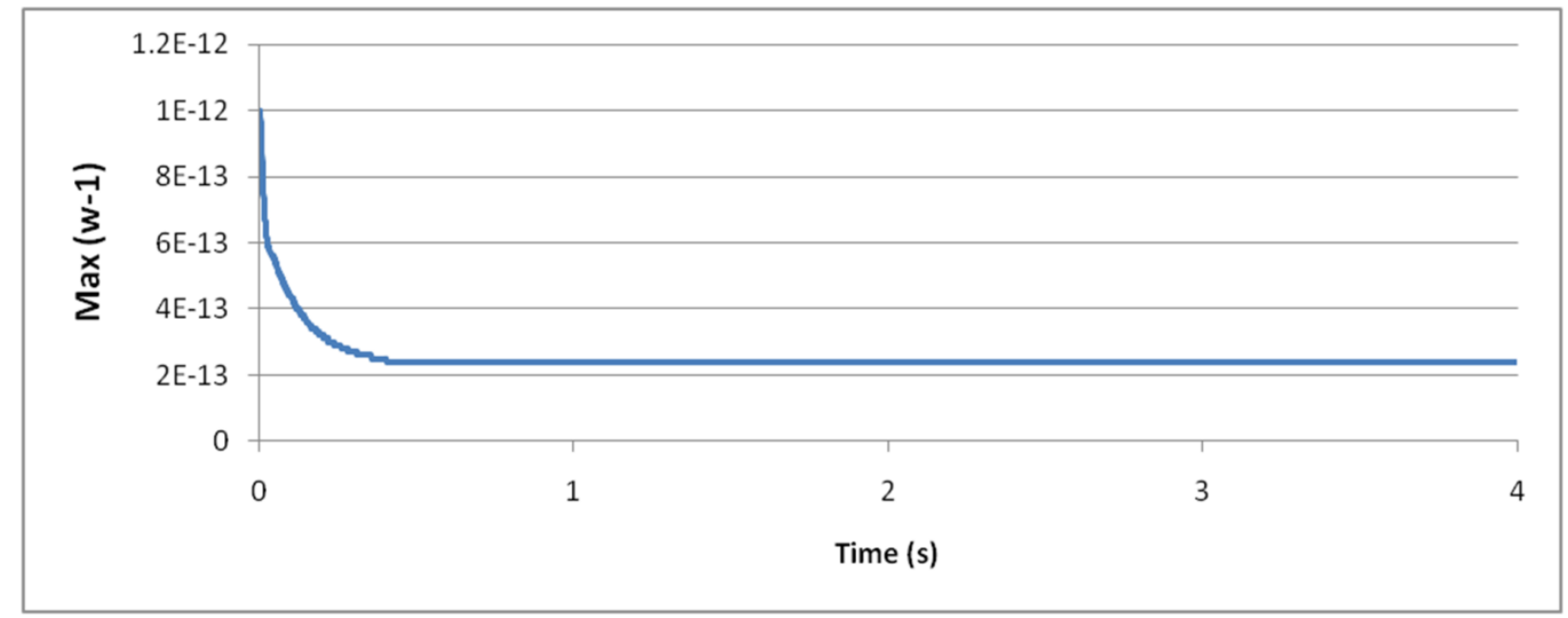

Fig. 5 - Example 2: $\max (w-1)$ as a function of $t$ for $\varepsilon=10^{-12}$.

Next, a larger perturbation height $\varepsilon=10^{-4}$, is selected. The 3D view of water surface elevation computed using the mesh with $6 \times 100 \times 100$ cells at $t=0.6,0.9,1.2,1.5$ and 1.8 is 
shown in Fig. 6. It can be seen that the water surface is well resolved and it is free of noises and spurious waves.

In order to show the importance of well-balanced property of the scheme, we apply a non-wellbalanced version of our scheme to the initial-boundary value problem with $\varepsilon=10^{-4}$. To obtain the non-well-balanced scheme, the proposed well-balanced quadratures (26) and (27) are replaced with a straightforward midpoint rule discretization:

$\bar{S}_{j}^{(2)}=-g\left(\overline{w_{J}}-B_{j}\right) B_{x}, \bar{S}_{j}^{(3)}=-g\left(\overline{w_{J}}-B_{j}\right) B_{y}$.

The non-well-balanced solution (water surface) computed at times $t=0.6,0.9,1.2,1.5$ and 1.8 on the same grid is shown in Fig. 7 (right column) and compared with the results obtained from the proposed well-balanced scheme (left column).
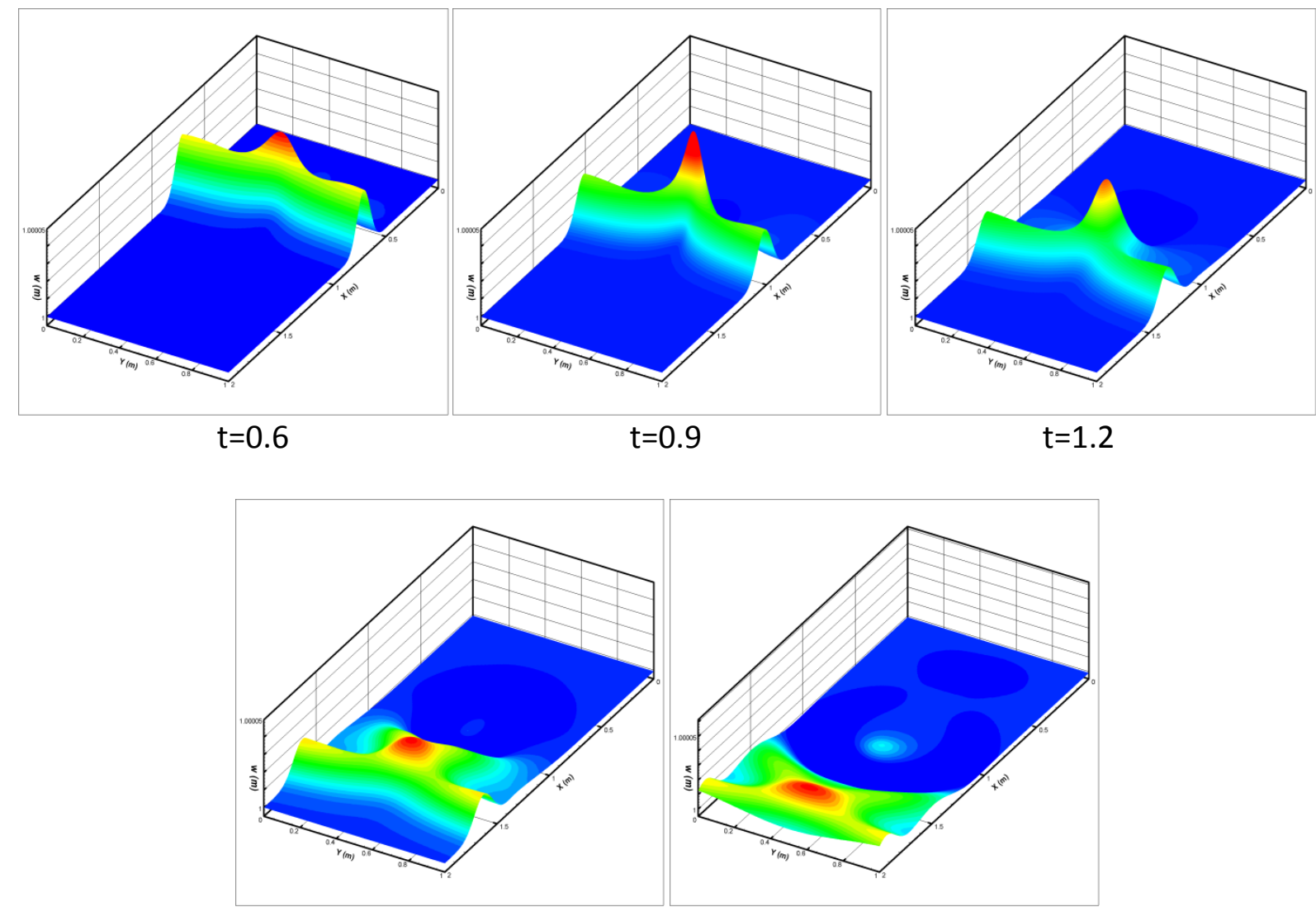
Fig. 6 - Example 2: 3D view of $w$ computed using the mesh with $6 \times 100 \times 100$ cells for

$$
\varepsilon=10^{-4} \text {. }
$$

One can see that in this case, even with the larger value of $\varepsilon$, there are spurious waves propagating in the domain and their magnitudes are close to the magnitude of the perturbation. This can evidently prove the advantage of the well-balanced proposed quadrature (28) used for source term discretization.
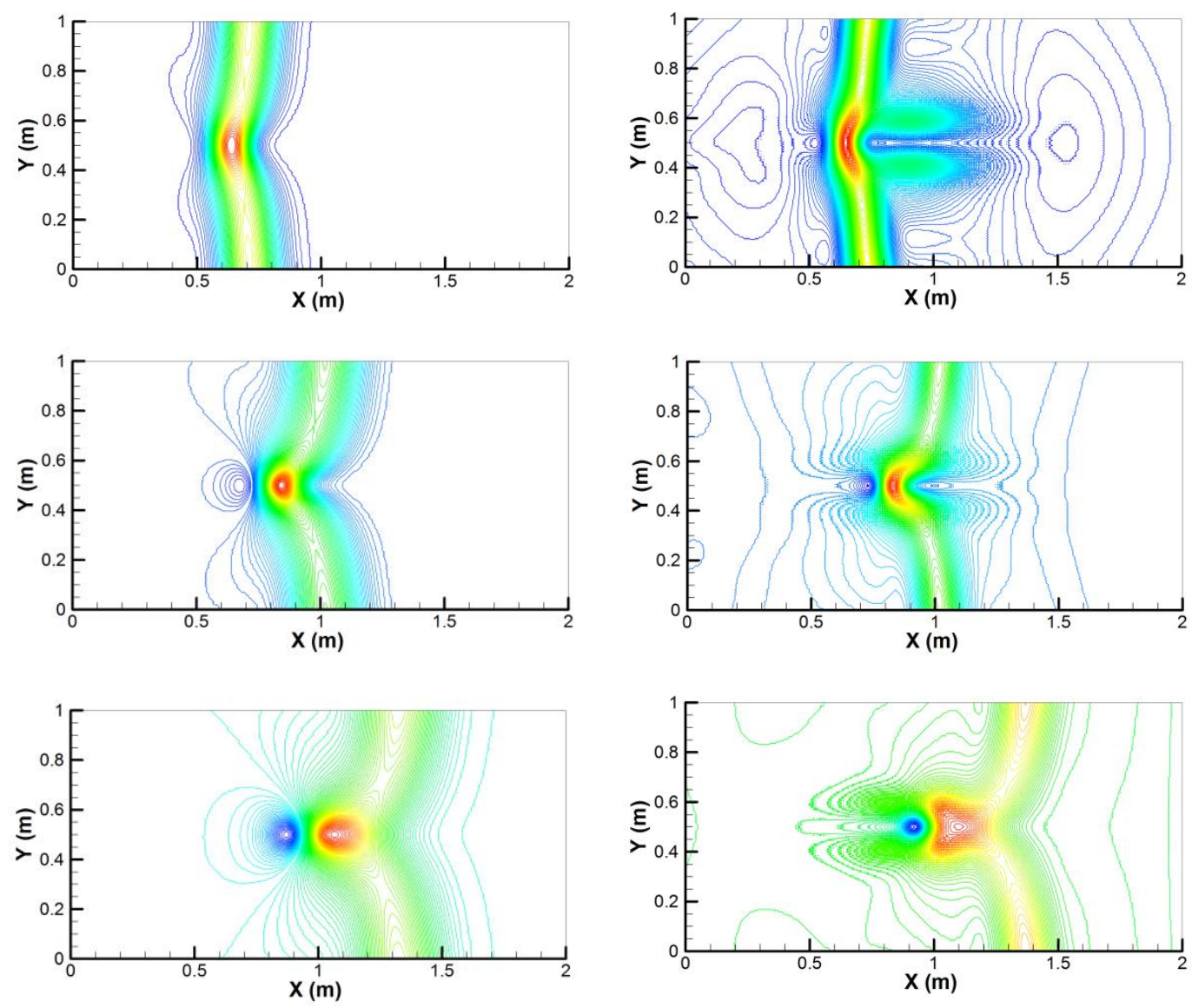

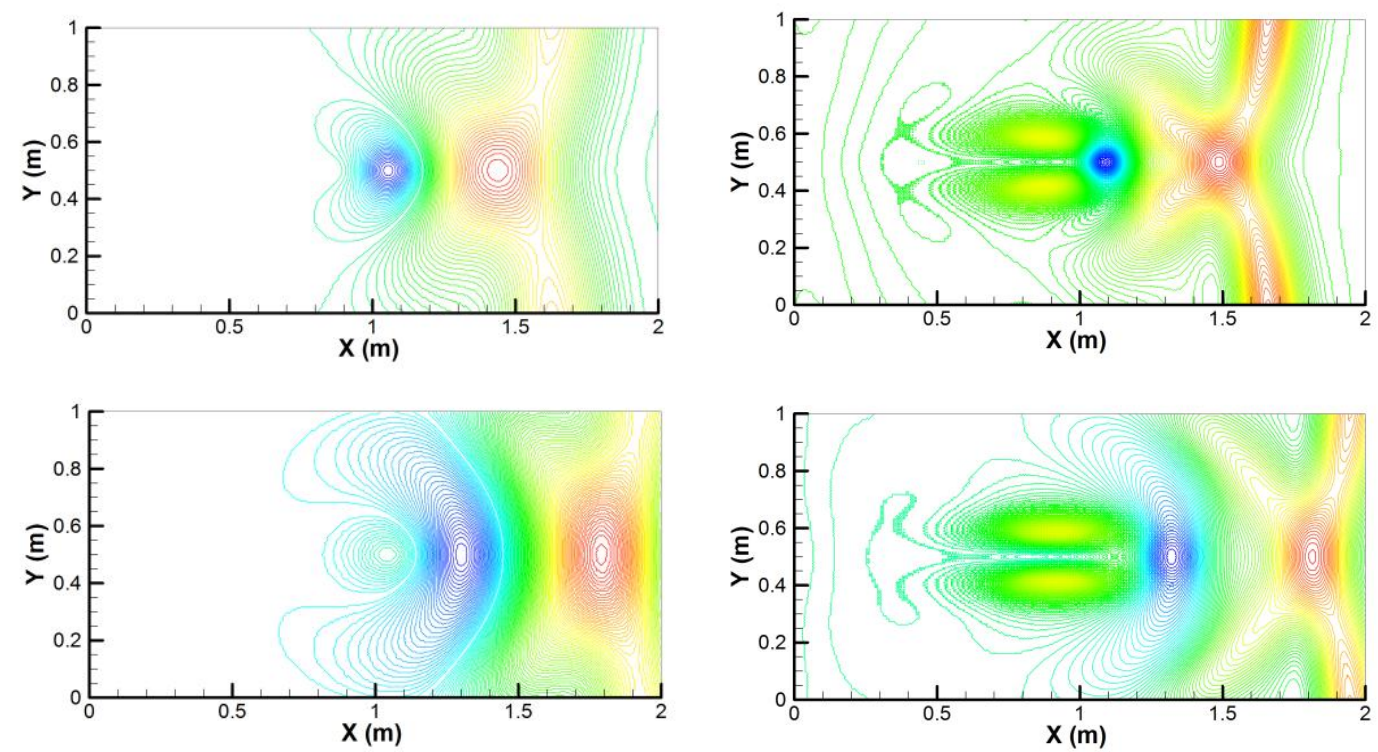

Fig. 7- Example 2: $w$ for $\varepsilon=10^{-4}$ computed using the well-balanced (left column) and nonwell-balanced (right column) schemes.

\section{Example 3 - Small Perturbation over Submerged Flat Plateau}

In this numerical test, taken from [23], we study the case of a submerged flat plateau as schematically shown in Fig. 8. Notice that in this example, the plateau is very close to the water surface, and that the initial water depth over the plateau is close to the perturbation height $\varepsilon=10^{-4}$. The computational domain is $[0,1] \times[0,1]$ and the bottom topography is given by $B(x, y)=\left\{\begin{array}{lr}1-2 \epsilon, & r \leq 0.1 \\ 10(1-2 \epsilon)(0.2-r), & 0.1 \leq r \leq 0.2 \\ 0, & \text { otherwise }\end{array}\right.$

where $r=\sqrt{(x-0.5)^{2}+(y-0.5)^{2}}$ and the initial data are:

$w(x, y, 0)=\left\{\begin{array}{cc}1+\epsilon, & 0.1 \leq x \leq 0.2, \\ 1, & \text { otherwise }\end{array} u(x, y, 0) \equiv v(x, y, 0)=0\right.$ 


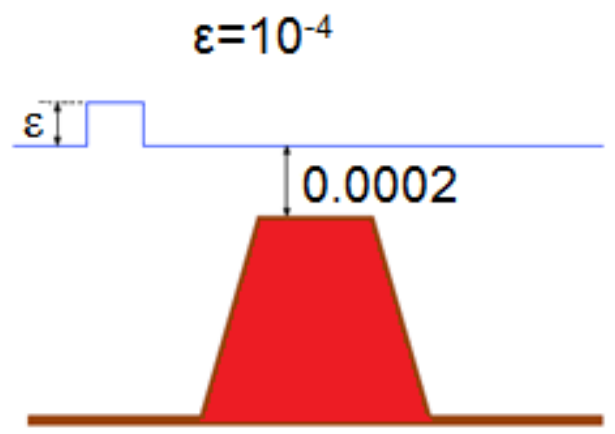

Fig. 8 - Example 3: 1D slice of bottom topography (39). The plot is not to scale.

The transparency boundary conditions are implemented at vertical boundaries of the domain while the horizontal boundaries are periodic. The solution is computed by the proposed wellbalanced central-upwind scheme using $6 \times 100 \times 100$ quadrilateral cells and the obtained water surface $w$ is plotted in Fig. 9 (left column) at $t=0.2,0.35,0.5$ and 0.65 . As one can observe, the general structure of the solution is well captured by the proposed method and there is no spurious wave propagating in the domain.

The water surface $w$ computed using the non well-balanced scheme on the same grid with $6 \times 100 \times 100$ cells is shown in Fig. 9 (right column).

As Fig. 9 shows, the spurious waves generated around the plateau dominate the solution. Comparing the results to those computed by the proposed well-balanced scheme, instructively shows the advantage of the well-balanced scheme. 

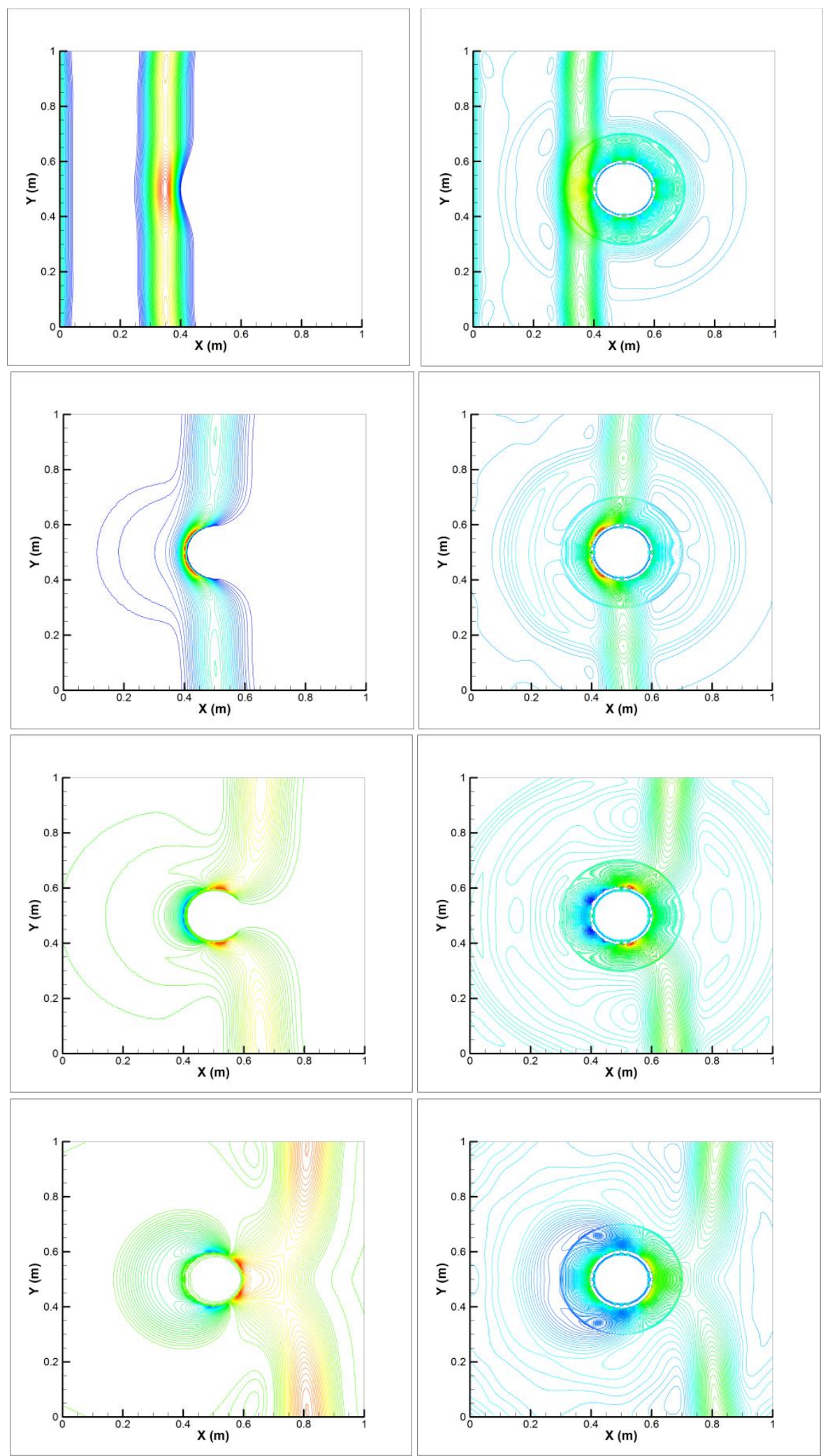

Fig. 9 - Example 3: $w$ computed by the proposed well-balanced (left) and non-well-balanced (right) schemes. 


\section{Example 4 - Small Perturbation Bending around a Round-Shape Island}

In this example, we examine both well-balanced and positivity preserving properties of the new scheme by testing its ability to handle a situation with a small perturbation of a "lake at rest" propagating around an island.

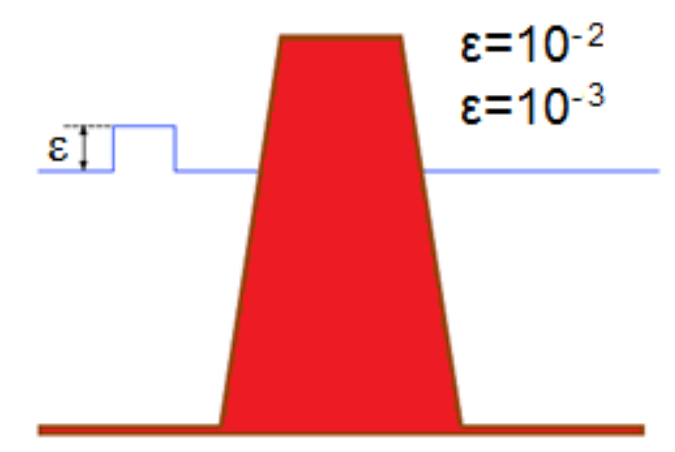

Fig. 10 - Example 4: 1D slice of the bottom topography (41). The plot is not to scale.

The bottom hump is above the water surface so that there is a disk-shaped island at the origin,

$B(r)=\left\{\begin{array}{lr}1.1, & r \leq 0.1 \\ 11(0.2-r), & 0.1<r<0.2 \\ 0, & \text { otherwise }\end{array}\right.$

see Fig. 10 . The computational domain is $[0,1] \times[0,1]$ and the initial conditions are given by

$w(x, y, 0)=\left\{\begin{array}{c}1+\epsilon, \quad 0.1<x<0.2, \\ \max \left\{1, B\left(\sqrt{x^{2}+y^{2}}\right)\right\}, \text { otherwise, }\end{array}, u(x, y, 0) \equiv v(x, y, 0)=0\right.$.

3D-view of the water surface $w$ computed at times $t=0.2,0.35,0.5$ and 0.65 using $6 \times 100 \times$ 100 cells with $\varepsilon=10^{-2}$ is shown in Fig. 11 where the right-going disturbance bends around the island. Though there are dry and almost dry cells which are changing their wet/dry status repetitively, the solution obtained by the proposed central-upwind scheme is non-oscillatory 
and well resolved. In order to make the experiment more challenging, we set $\varepsilon=10^{-3}$. The water surface $w$ computed at times $t=0.2,0.35,0.5$ and 0.65 using $-6 \times 100 \times 100$ cells is presented in Fig. 12 (left column). To test the numerical convergence of the proposed method, we refine the mesh and use $6 \times 200 \times 200$ cells. The obtained results, plotted in Fig. 12 (right column), demonstrate nice convergence property of the developed central-upwind scheme.
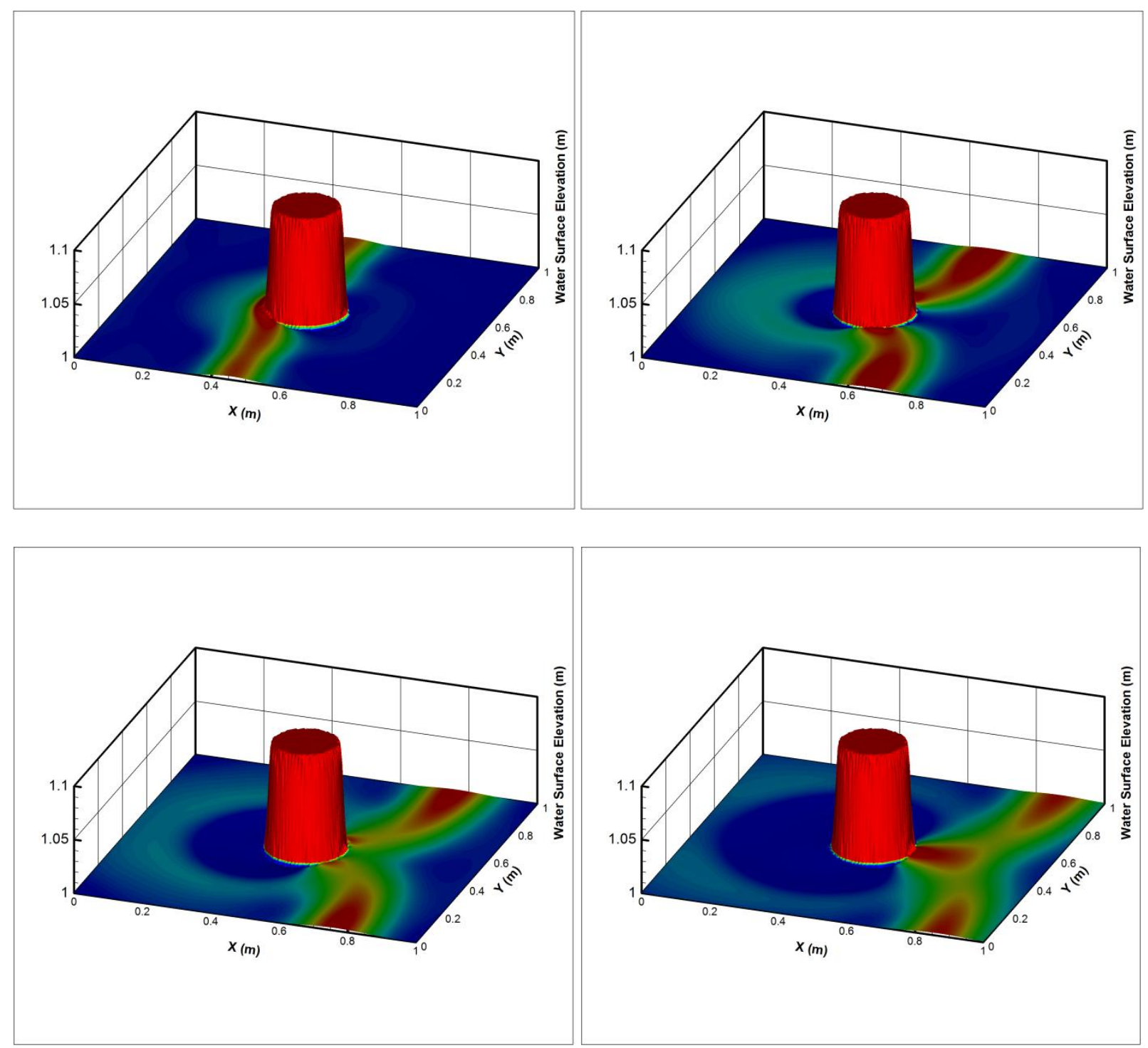

Fig. 11 - Example 4: 3D view of $w$ for $\varepsilon=10^{-2}$ computed using $6 \times 100 \times 100$ quadrilaterals 

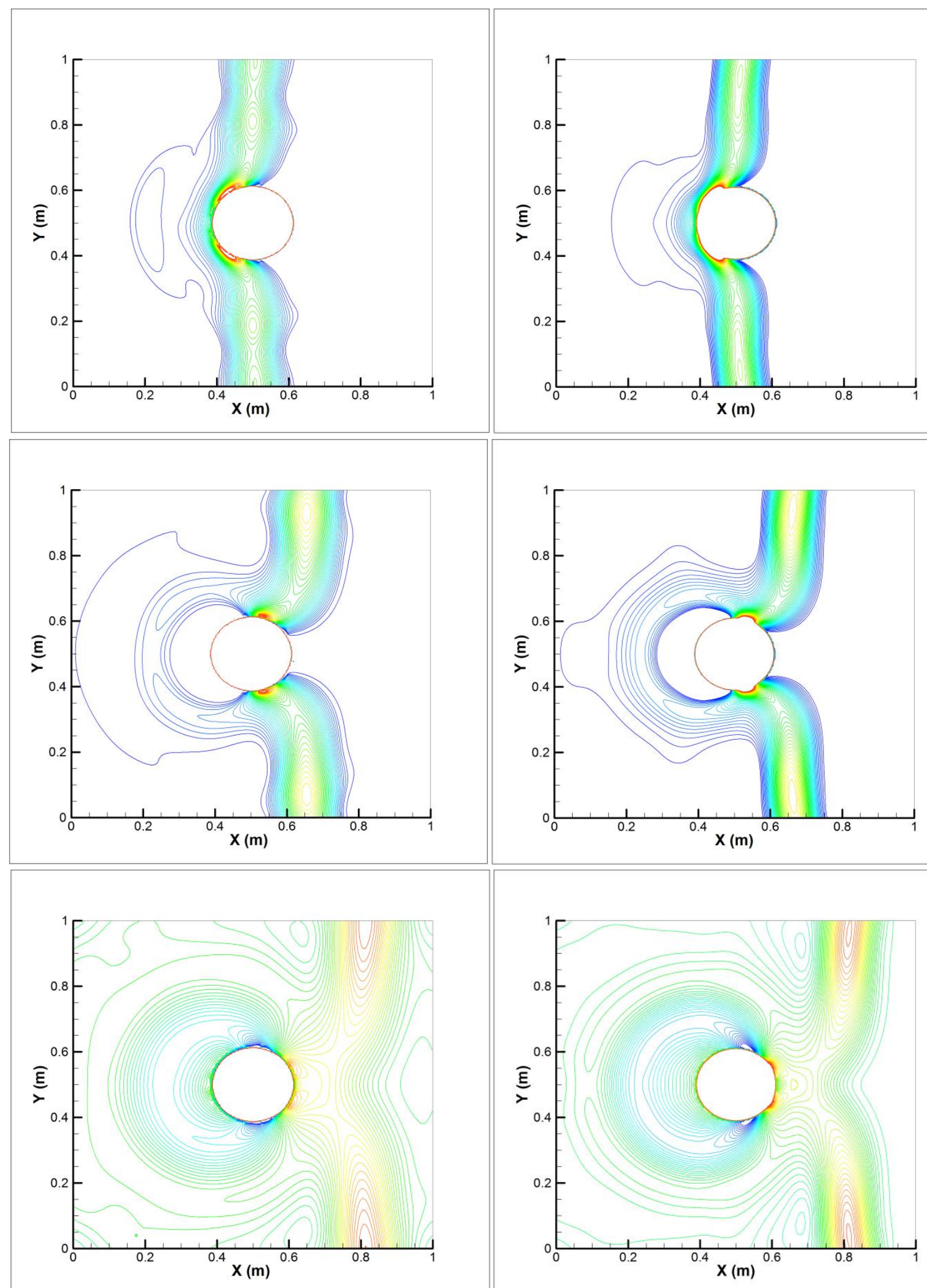

Fig. 12 - Example 4: $w$ with $\varepsilon=10^{-3}$ computed using the grids with $6 \times 100 \times 100$ (left column) and $6 \times 200 \times 200$ (right columns) cells. 


\section{Example 5 - Dam Break over Discontinuous Bottom Topography}

In the final example, we not only verify the ability and robustness of the proposed centralupwind scheme on the quadrilateral grid, but also demonstrate an advantage of the proposed scheme over the triangular central-upwind scheme from [23]. The computational domain is $[-4,4] \times[-4,4]$ and a cylindrical dam is assumed to be placed on a dry island with the following bottom topography:

$B(x, y)=\left\{\begin{array}{lr}1, & |x|+|y| \leq 2, \\ 0, & \text { otherwise }\end{array}\right.$

The water is initially at rest $(u(x, y, 0) \equiv v(x, y, 0) \equiv 0)$ and the water level is given by

$$
w(x, y, 0)=\left\{\begin{array}{rr}
9, & x^{2}+y^{2} \leq 1 \\
1, & x^{2}+y^{2}>1 \text { and }|x|+|y| \leq 2 \\
0, & \text { otherwise }
\end{array}\right.
$$

The initial setting is shown in Fig. 13.

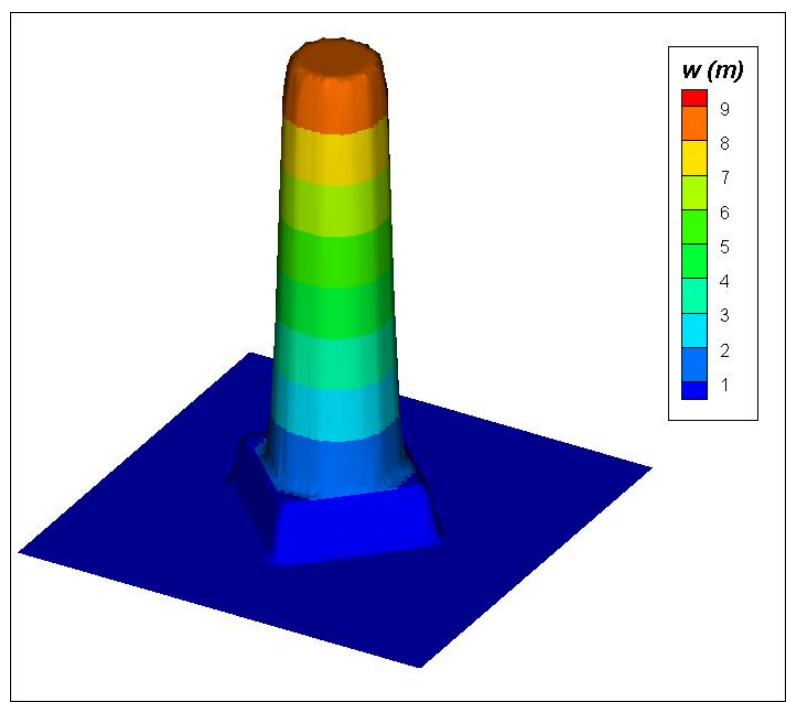

Fig. 13 - Example 5: 3D view of the bottom topography (43) and initial water surface (44). 
The dam is getting broken at time $t=0$. The flow structure after the dam break is very complex. In order to make a proper comparison between the proposed scheme on the quadrilateral grid and the triangular central-upwind scheme, we keep the number of cells (cell areas) equal in both cases. In Fig. 14, we plot 1D cross sections of the computed solutions at $y=0$. The water surface $w$ component of the solution computed at $t=0.6$ by the new scheme using $6 \times 50 \times 50$ quadrilateral cells and by the triangular central-upwind scheme using $4 \times 62 \times 62$ triangular cells are shown in Fig. 14 (left). One can clearly see that the water surface structure is resolved more accurately by the proposed quadrilateral central-upwind scheme. We then refine the mesh and use $6 \times 100 \times 100$ quadrilaterals versus $4 \times 124 \times 124$ triangular cells. The finer results are presented in Fig. 14 (right). Evidently, even with a finer mesh, the structure of solution is better resolved by the proposed scheme.

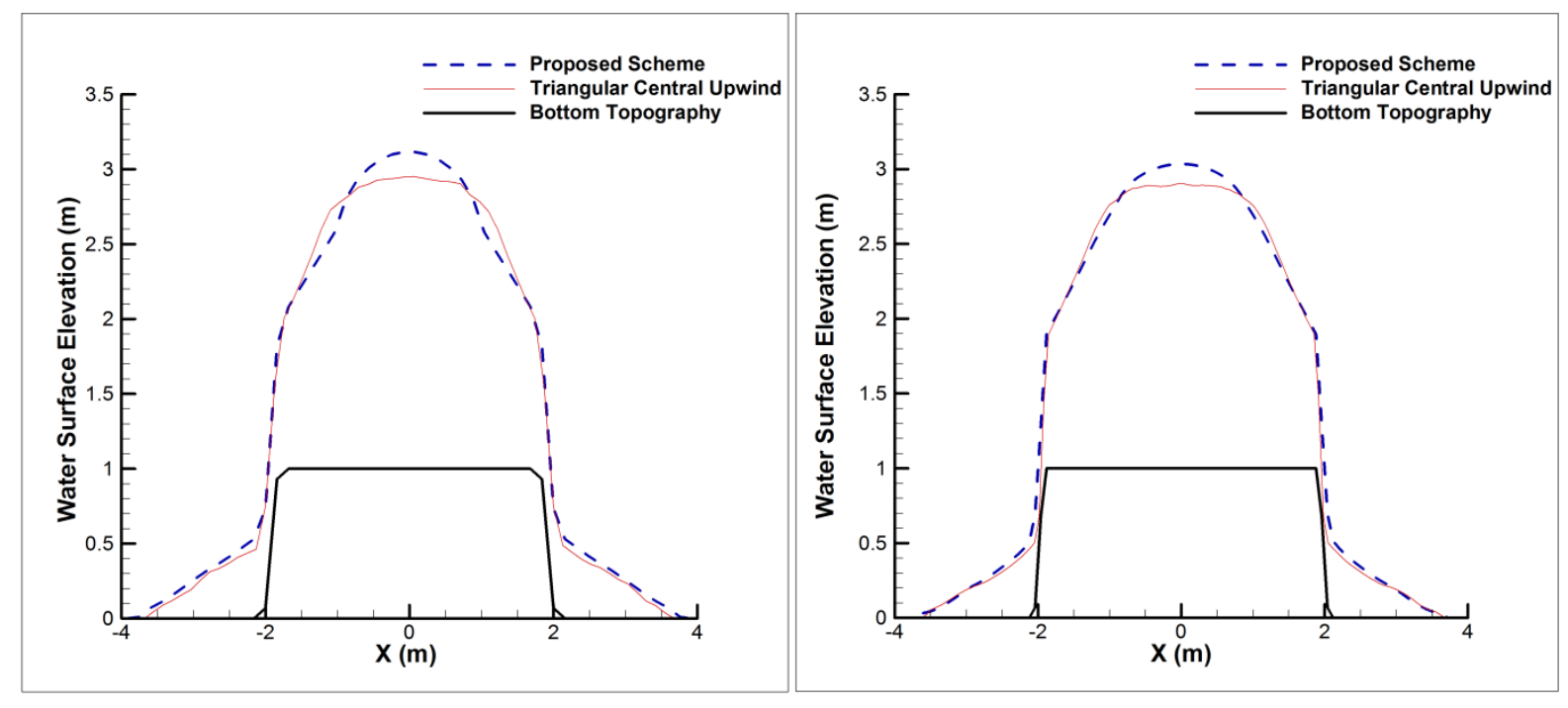

Fig. 14 - Example 5: 1D slices of $w$ computed using the proposed quadrilateral and triangular central-upwind schemes using coarser (left) and finer (right) grids. 
The contour lines of the water surface $w$ computed at $t=0.6$ using the finer grids are presented in Fig. 15. One can clearly observe that the proposed scheme on a quadrilateral grid has captured and resolved the complex flow patterns, both over and around the island, with a significantly higher resolution. In addition, some fluctuations can be observed in the water surface computed by the triangular scheme, while the one obtained by quadrilateral scheme is well resolved and free of fluctuations.
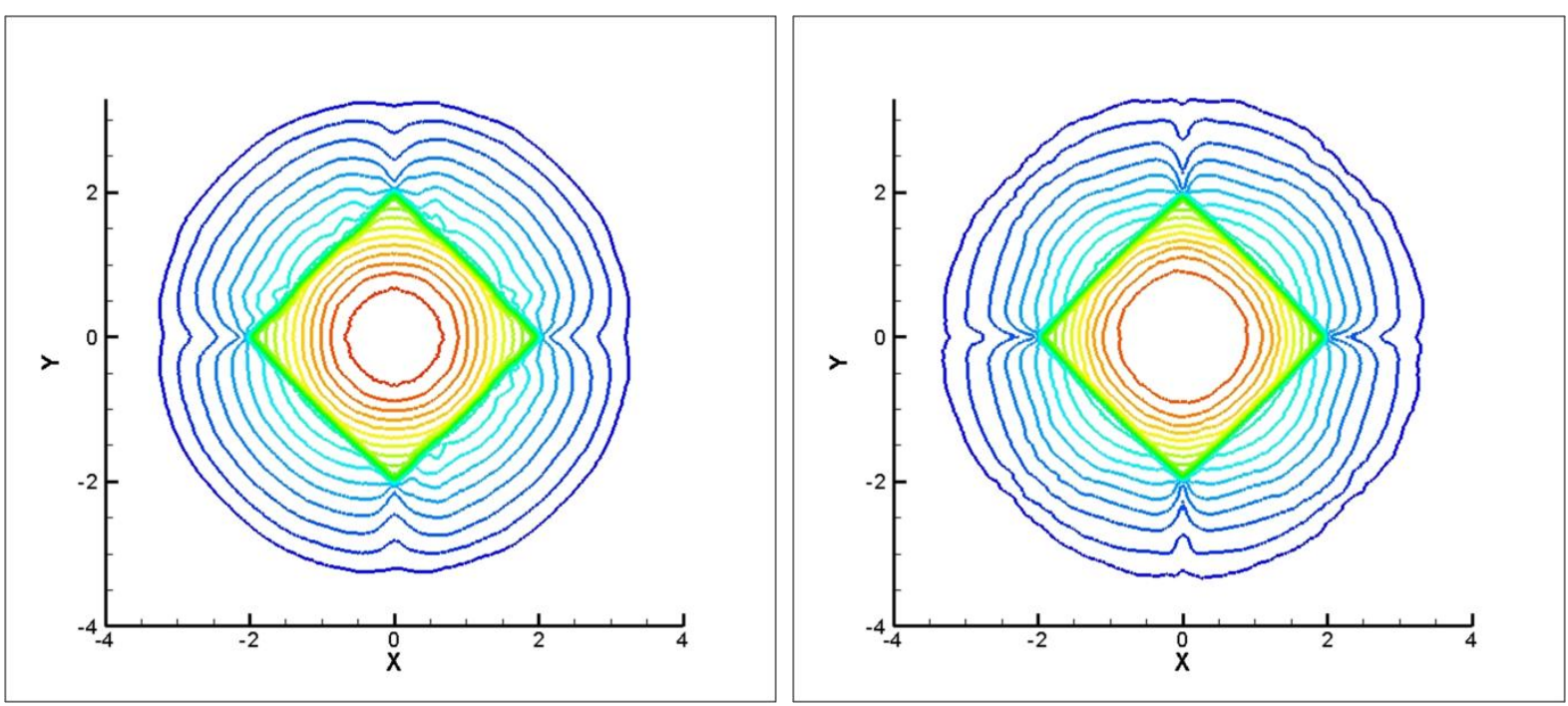

Fig. 15 - Example 5: Contour plots of $w$ at $t=0.6$ using the proposed quadrilateral (left) and triangular (right) central-upwind schemes.

The velocity ( $u$ and $v$ ) components of the solution computed at $t=0.6$ using both the coarser and finer grids are also presented in Fig. 16. One can clearly observe that although $u$ computed by both schemes are quite close, the velocity computed by the proposed method is smoother and the velocity spark over the island is much lower (Fig. 16a). However, by refining the mesh the results become closer (Fig. 16b). For the $v$ component of the solution, one can see that the 
results obtained using the triangular grid is noisy, while those computed by the quadrilateral grid are completely smooth and free of oscillations and fluctuations (Fig. 16c). In addition, one can see that even by refining the mesh, the results of the triangular central-upwind scheme keep fluctuating (Fig. 16d).

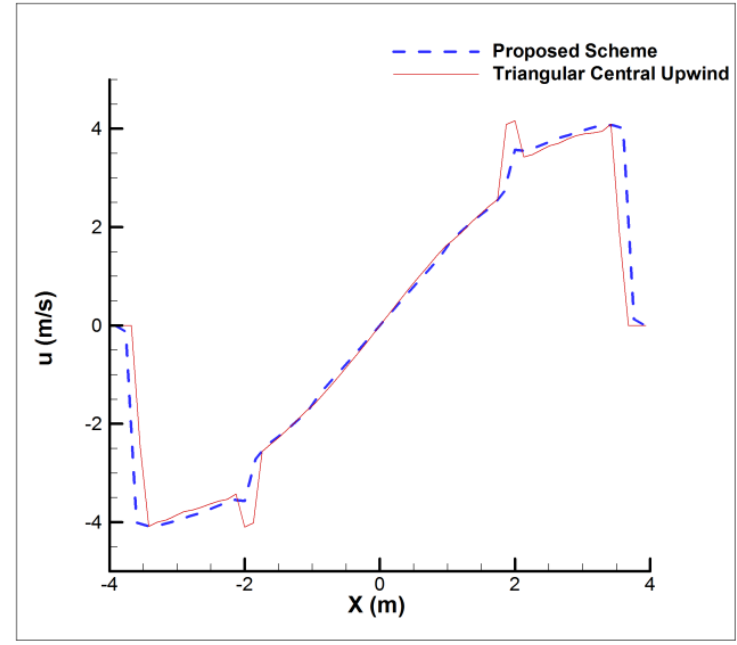

(a)

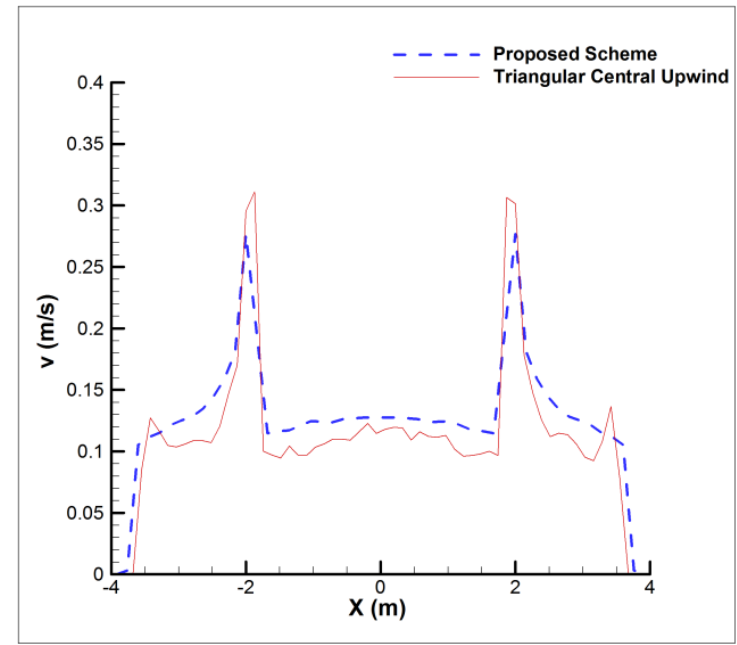

(c)

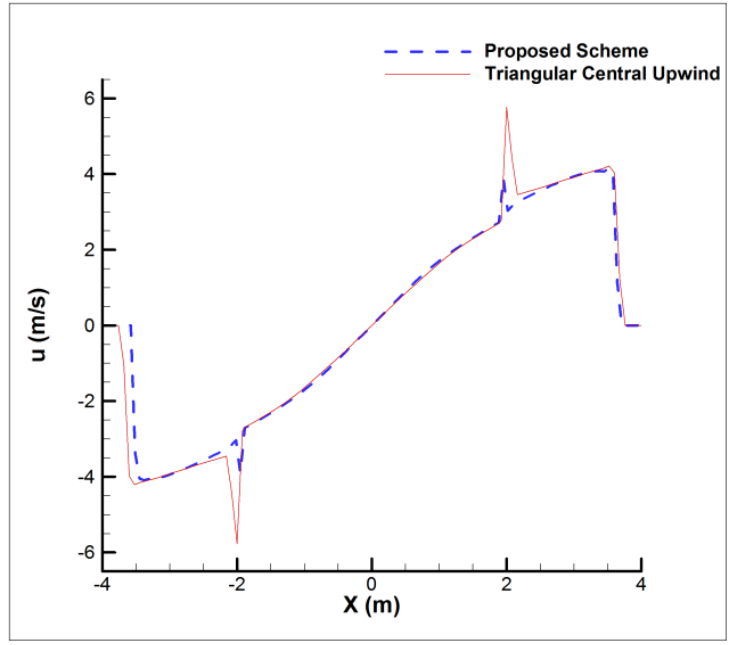

(b)

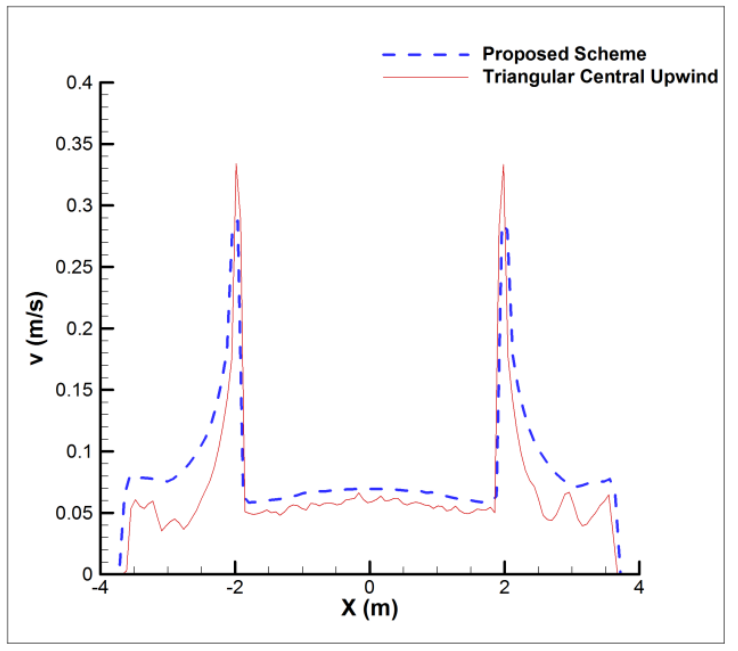

(d)

Fig. 16 - Example 5: $1 \mathrm{D}$ slices of $u$ computed using (a) the coarser grids with $6 \times 50 \times 50$ quadrilateral cells $(4 \times 62 \times 62$ triangular cells); (b) finer grids with $6 \times 100 \times 100$ quadrilateral cells $(4 \times 124 \times 124$ triangular cells); and $v$ computed using $(c)$ the coarser and (d) finer grids. 
Finally, in Fig. 17, the time evolution of the $v$ component of the solution computed on the finer grids is illustrated at $t=0.2,0.3,0.4,0.5$ for both schemes. It clearly shows that the proposed scheme has a better performance in predicting the velocity. The results of time evolution of $v$ component of the solution (not shown here) also confirm that the velocity field computed by the triangular central-upwind scheme is noisy, while the one computed by the developed scheme on the quadrilateral grid is quite smooth and oscillation-free.

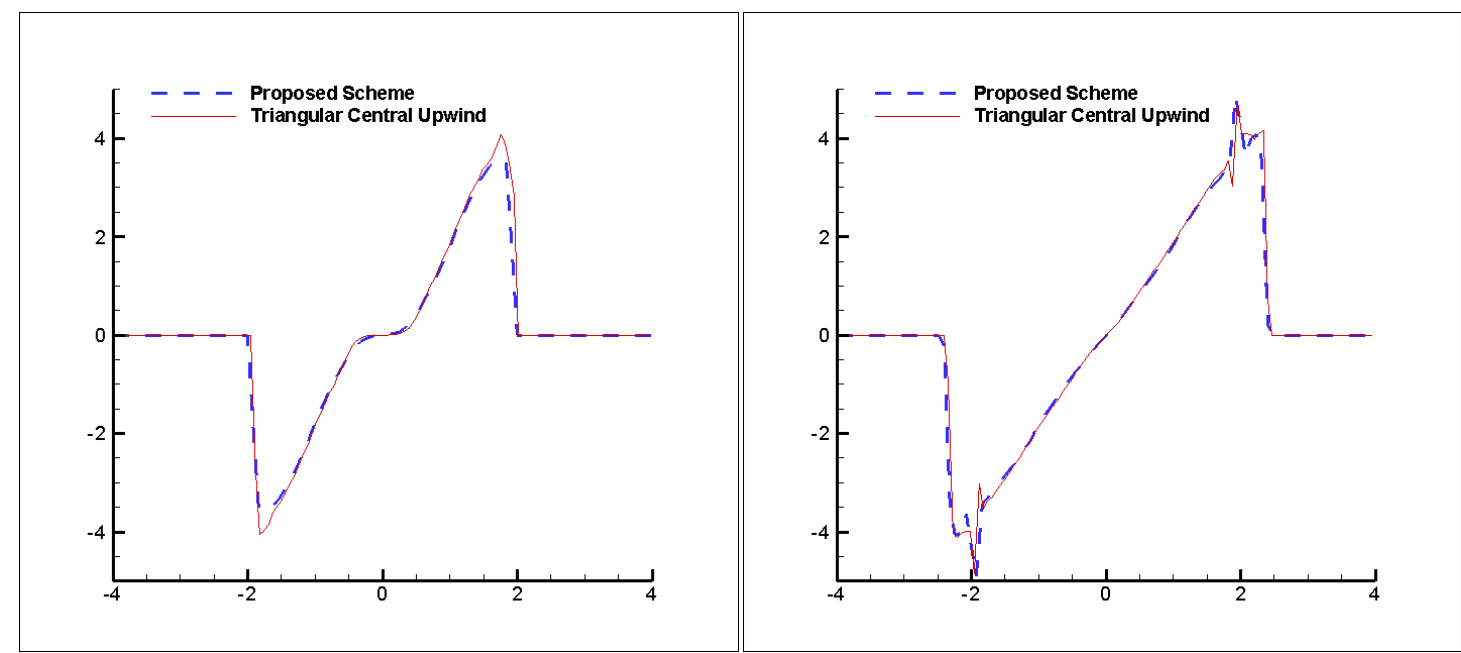

(a)

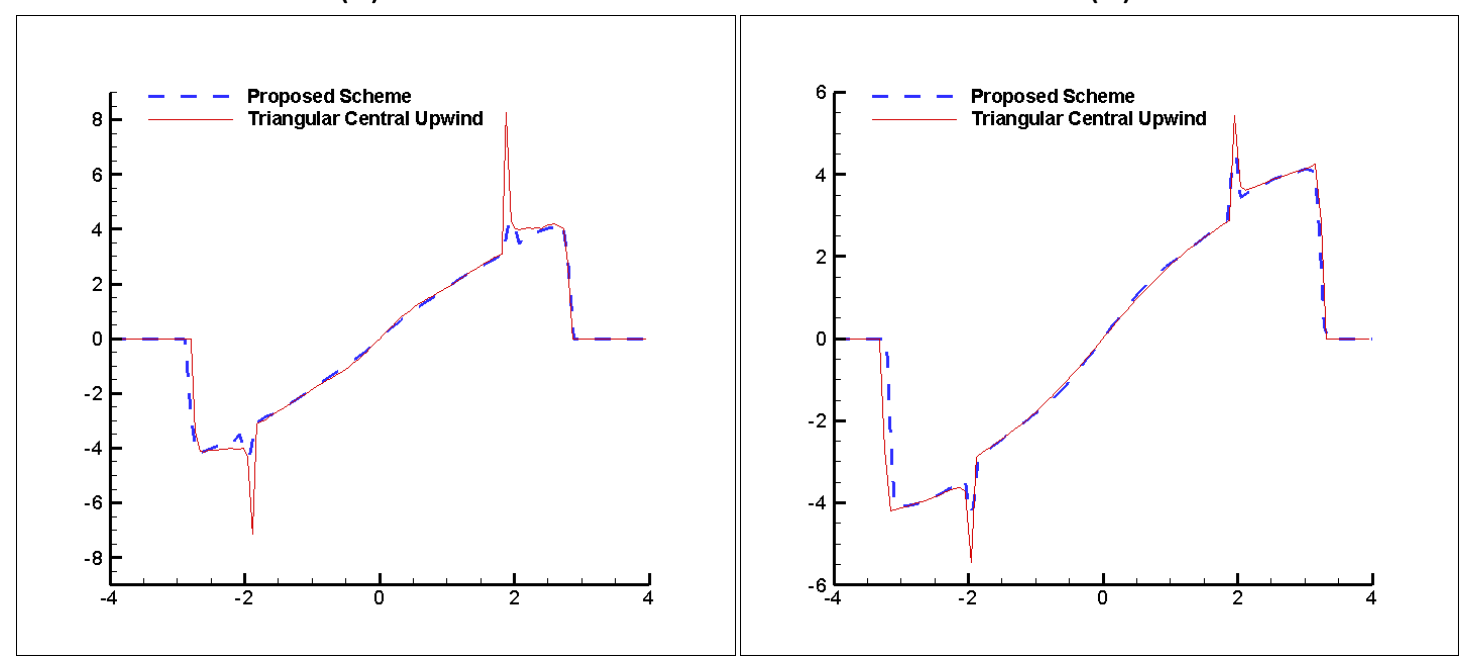

(c)

(d)

Fig. 17 - Example 5: Comparison of $u$ (1D slices along $y=0$ ) computed using the finer grids at (a) $t=0.2$, (b) $t=0.3$, (c) $t=0.4$ and (d) $t=0.5$. 


\section{Example 6 - Steady flow over a bump}

In order to demonstrate the performance of the proposed method in modelling steady flows, we consider a steady flow over a bump test which has been widely used in the literature; see e.g., $[2,41]$. We consider a flume with the following topography:

$B(x, y)= \begin{cases}0.2-0.05(x-10)^{2}, & 8<x<12 \\ 0, & \text { otherwise } .\end{cases}$

In order to have a Transcritical flow with a shock, fixed boundary conditions for the water depth $\mathrm{h}=0.33 \mathrm{~m}$ and discharge $\mathrm{uh}=0.18 \frac{\mathrm{m}^{2}}{\mathrm{~s}}$ are imposed at the downstream $(x=22 \mathrm{~m})$ and upstream $(x=0)$ boundaries, respectively. The analytical solution of this problem is given in [42]. The water surface $w$ and discharge $u h$ computed by the proposed scheme using $6 \times 8 \times 88$ quadrilateral cells are compared with the exact solutions in Fig. 18 . As one can see, the computed solution is in good agreements with the analytical one except for some small numerical oscillations. It should be pointed out that similar oscillations have been also reported in most existing numerical models; see e.g., $[2,41,43,44]$.

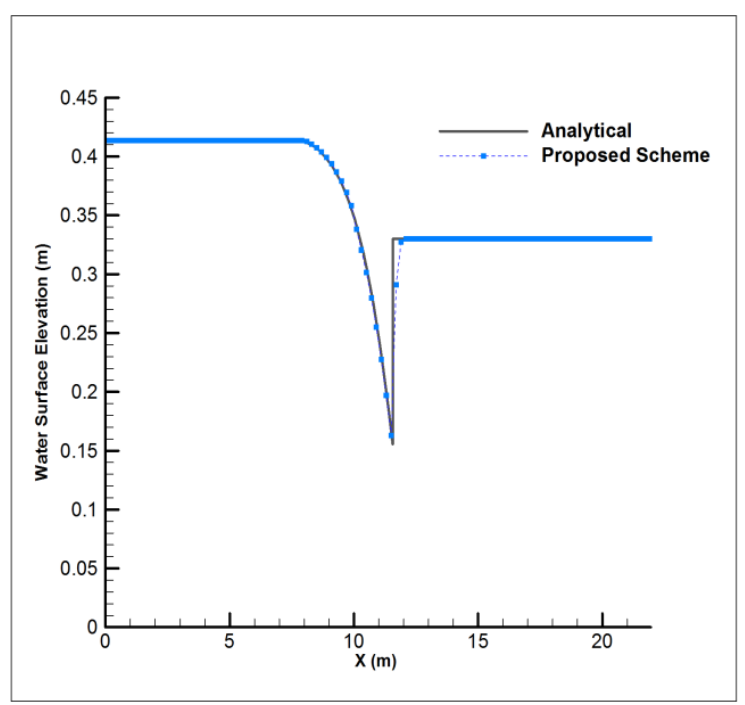




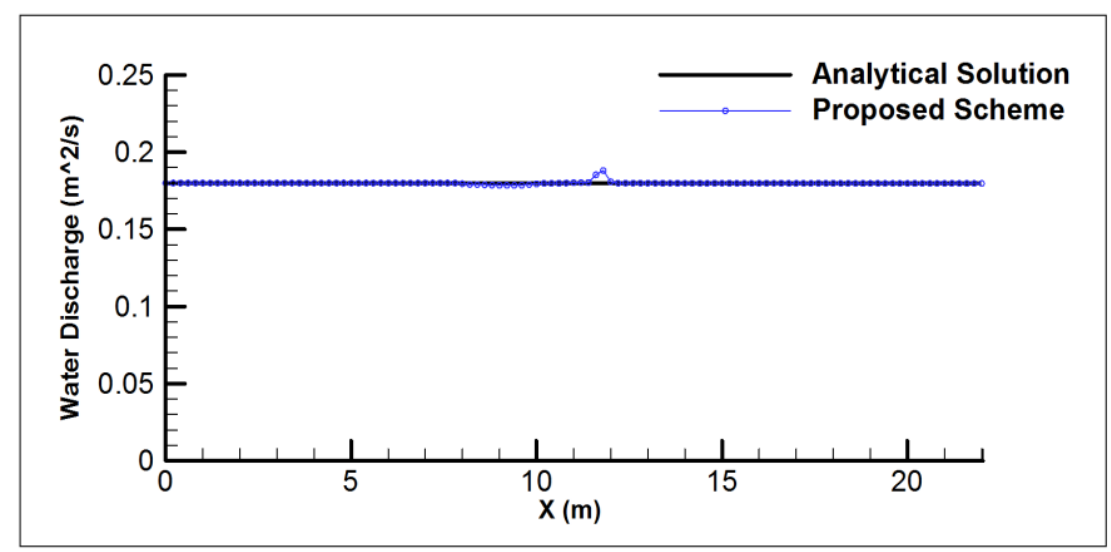

Fig. 18 - Example 6: Steady transcritical flow over a bump with a shock: water surface elevation (top) and discharge (bottom).

\section{Example 7 - 2D dam break simulation}

In this example, we examine the performance of the proposed model in simulating a 2D dam break with complex geometry. The laboratory measurements for this experiments were reported in [45]. The dam break was carried out in a $21.2 \mathrm{~m}$ long flume with slope of 0.01 . The plan view of the flume can be seen in Fig. 19, where the fully unstructured quadrilateral mesh is also shown. As one can see, a converging-diverging formation creates 2D effects. The manning roughness coefficient of the flume is $n=0.012$ and we estimate the bed friction in $x$ - and $y-\quad$ directions $\quad$ using $\quad g n^{2}\left(\frac{\sqrt{2} h}{\sqrt{h^{4}+\max \left(h^{4}, \varepsilon\right)}}\right)^{\frac{7}{3}} \sqrt{(h u)^{2}+(h v)^{2}}(h u) \quad$ and $g n^{2}\left(\frac{\sqrt{2} h}{\sqrt{h^{4}+\max \left(h^{4}, \varepsilon\right)}}\right)^{\frac{7}{3}} \sqrt{(h u)^{2}+(h v)^{2}}(h v)$, respectively. Note that here the $1 / h$ term is approximated using the same desingularization procedure used for velocity calculation in (20). 


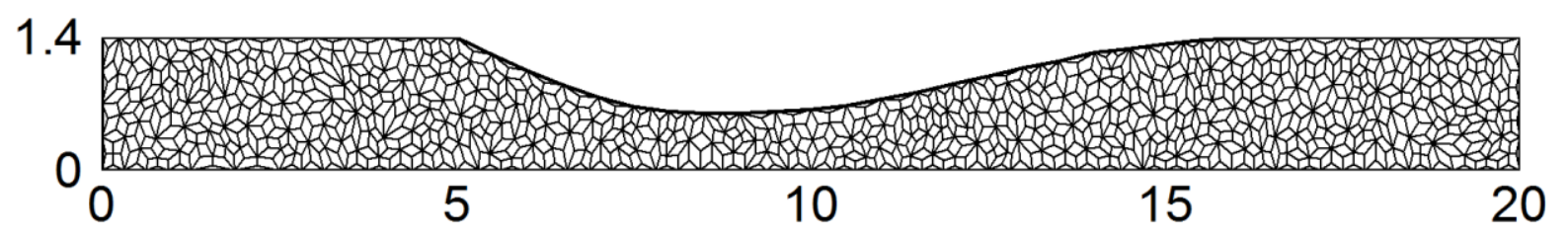

Fig. 19 - Example 7: Plan view of the flume together with the unstructured quadrilateral mesh.

With the water depth of $0.15 \mathrm{~m}$ at the dam, the computed water depth profiles at times $t=2.0 \mathrm{~s}, t=6.0 \mathrm{~s}$ and $t=20.0 \mathrm{~s}$ are compared to the measurements in Fig. 20.

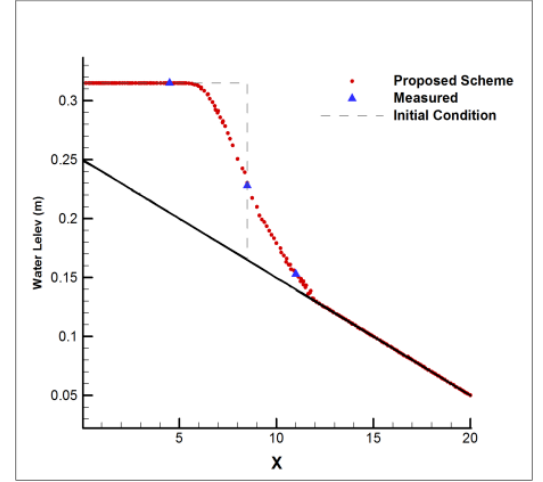

(a)

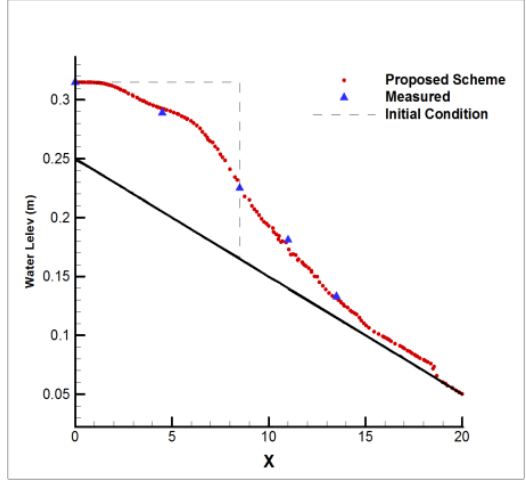

(b)

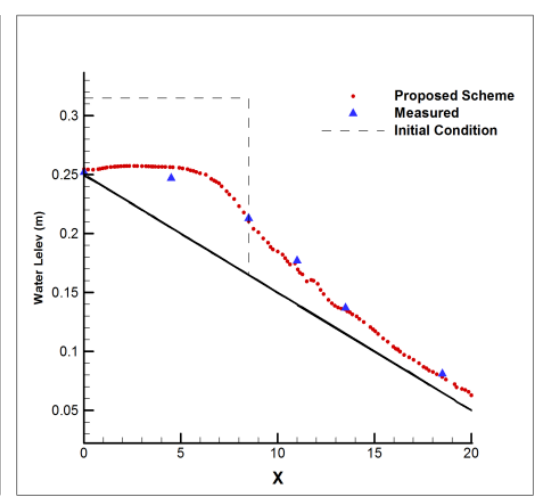

(c)

Fig. 20 - Example 7: Comparison between computed and measured water surface profiles at (a)

$$
t=2 s, \text { (b) } t=6 s \text { and (c) } t=20 s
$$

As one can see, the water surface profile is strongly affected by the $2 \mathrm{D}$ shape of the flume and the wave speed increases due to the sloping bed. For an initially dry bed, the proposed scheme performs well and the predicted water surface profiles are satisfactory.

\section{Example 8 - Dam break and advance over a triangular obstacle}

In this test case, we examine the performance of the proposed scheme with a dam break wave over a triangular obstacle at the downstream of the dam. The reservoir, channel and triangular obstacle geometry along with the location of the measurement gauges are presented in Fig. 21. The reservoir with initial water depth of $0.75 \mathrm{~m}$ is connected to a rectangular channel with a 
symmetric triangular obstacle located downstream of the dam. The Manning roughness coefficient is $n=0.0125$ for the bed. The boundaries are solid walls except for the free outlet at the end of the channel.

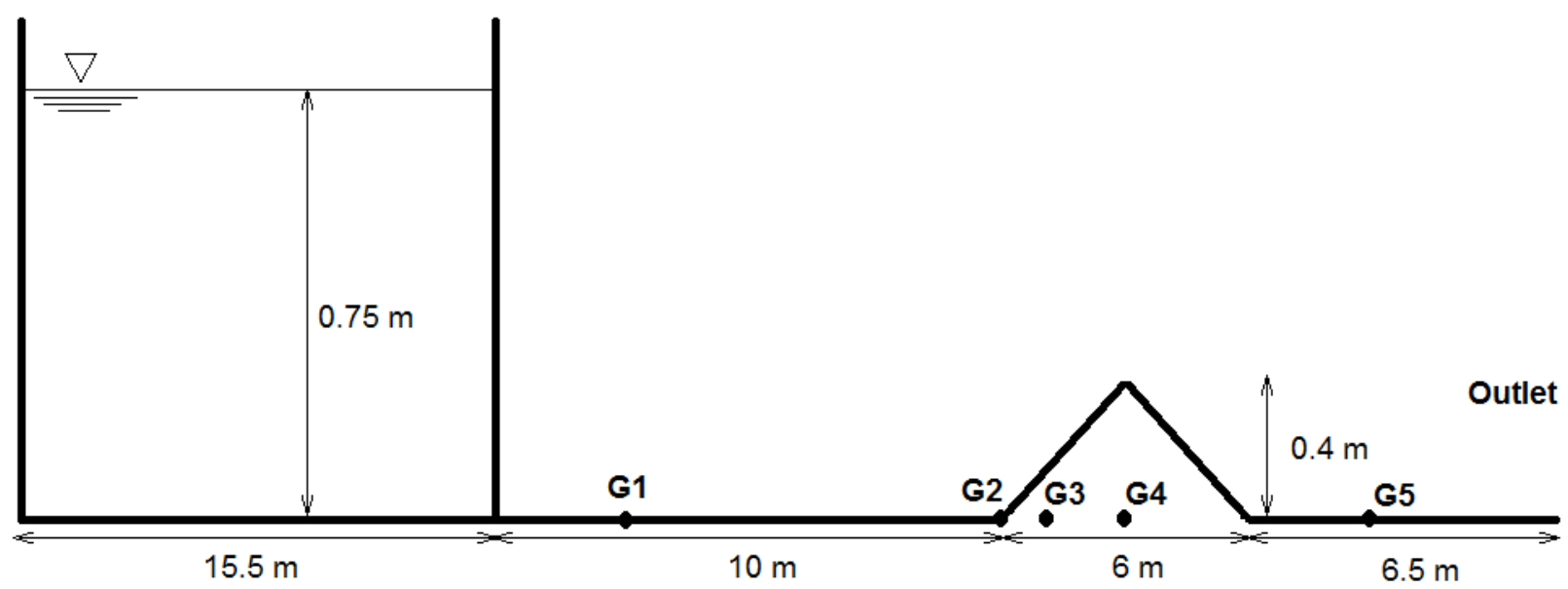

Fig. 21 - Example 8: Schematic side view of the experimental model and gauge point locations

The experimental data are reported in [46] for the gauge points G1, G2, G3, G4, and G5 which are located at $4 \mathrm{~m}, 10 \mathrm{~m}, 11 \mathrm{~m}, 13 \mathrm{~m}$ and $20 \mathrm{~m}$ downstream of the reservoir, respectively. We compute the solution using a quadrilateral mesh with The computed evolutions of water depths using a quadrilateral mesh grid with $6 \times 100 \times 8$ cells. In Fig. 22, we present the time evolution of the computed water depths at the gauge points and compare the obtained results with the measured values. For the Gauges G1, G2 and G3 which are located before the obstacle vertex, the water depth and the arrival time of the wave are well predicted. In addition, for Gauge G4 with a crucial location at the obstacle vertex, the wet/dry conversion is correctly predicted. At the Gauge G5, however, where the amount of water is not considerable a little disagreement is observed. It may result from vertical non-hydrostatic motions which are not considered in SWEs. The same feature is also reported in $[41,46,47]$. 


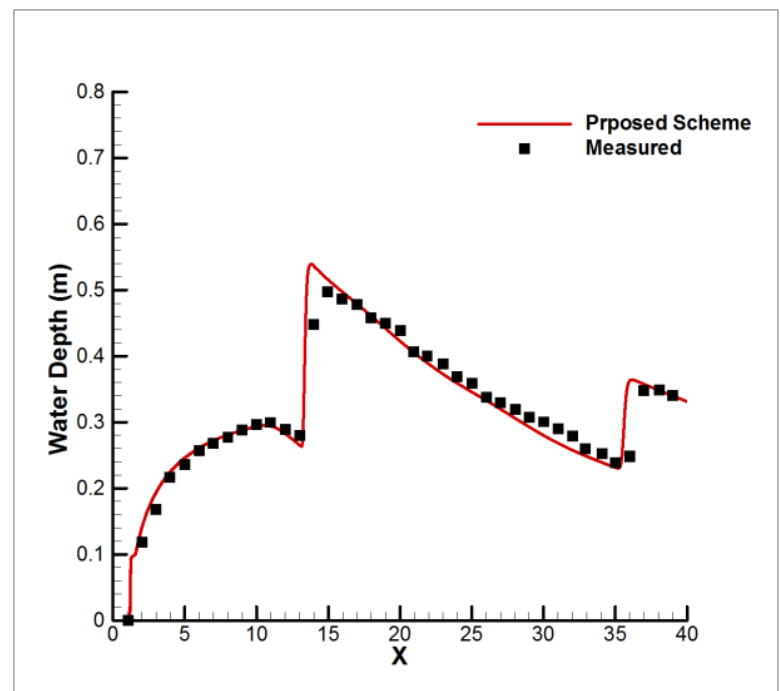

(G1)

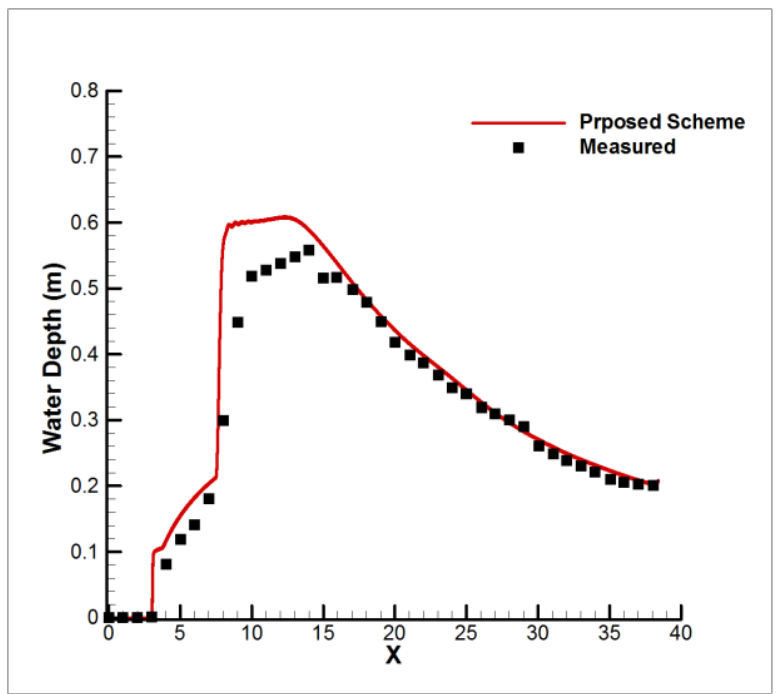

(G2)

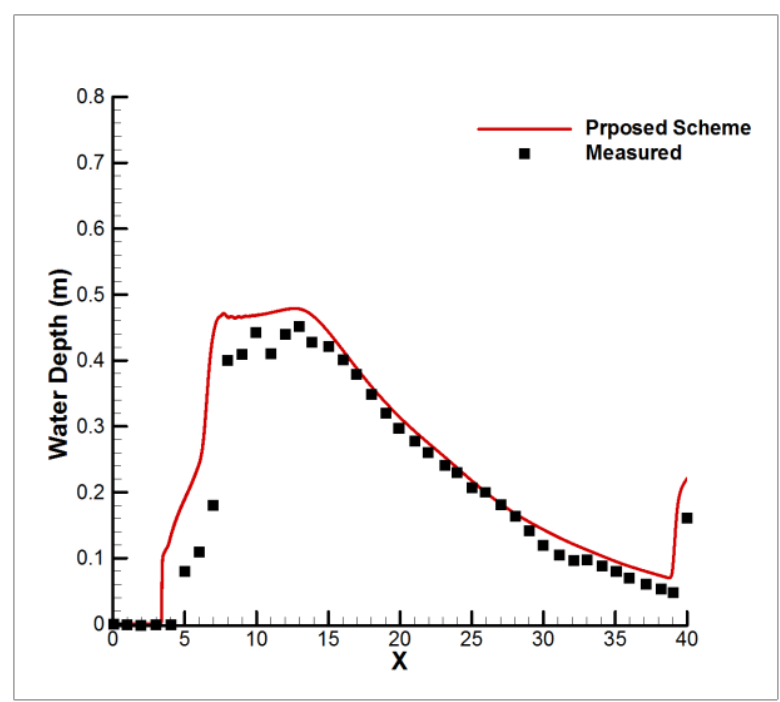

(G3)

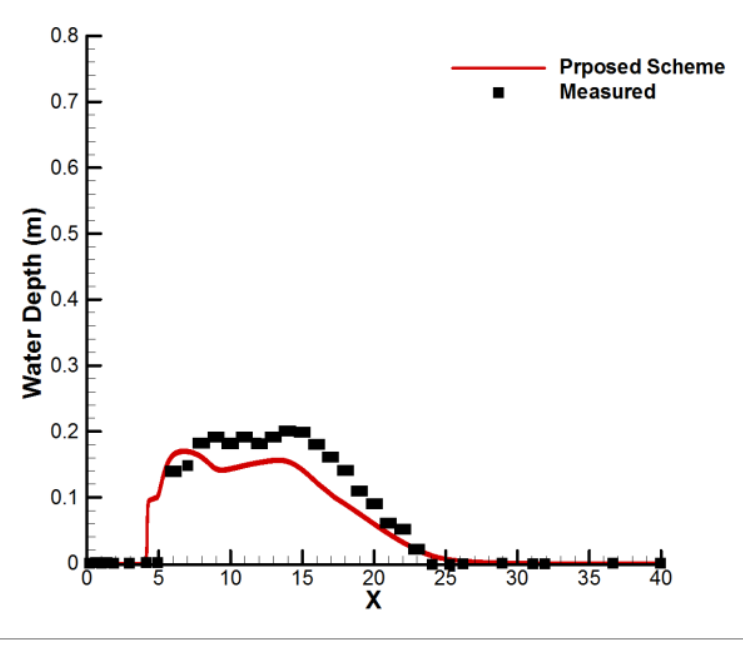

(G4) 


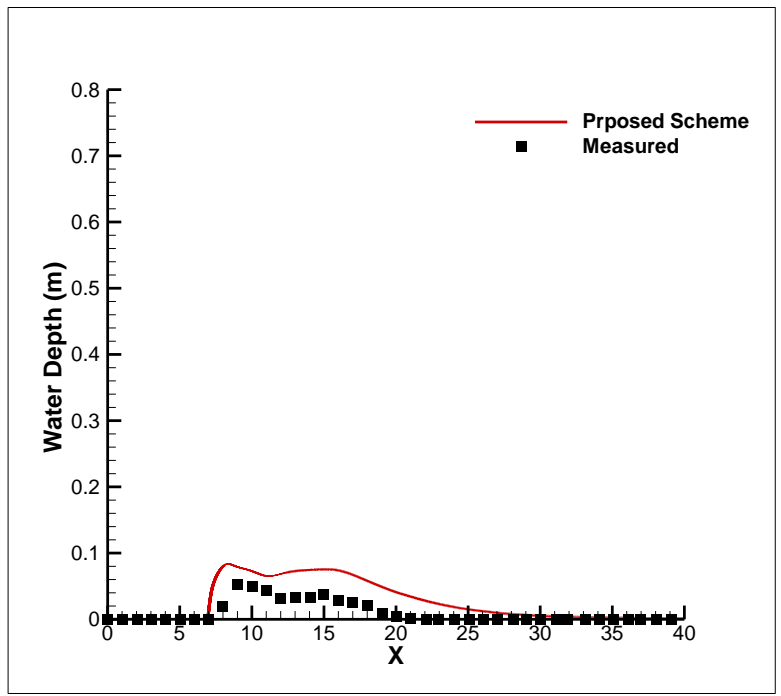

(G5)

Fig. 22 - Example 8: Comparison of the computed water depth with the corresponding measured values at the gauge points during the first $40 \mathrm{~s}$.

\section{Conclusion}

A new second-order central-upwind scheme for shallow water equations on the unstructured quadrilateral grids has been developed. To this end, we have proposed a new technique for bottom topography approximation over quadrilateral cells as well as an efficient water surface correction procedure. Moreover, we have designed a new quadrature for discretization of the source term. The proposed scheme guarantees the positivity of the computed fluid depth and exactly preserves "lake at rest" steady states. The proposed scheme on unstructured quadrilateral grids has several advantages over the triangular central-upwind scheme from [23]. For instance, such grid uses more information from the neighbouring cells in time evolution of the cell average values. We applied the proposed scheme to various numerical tests which exhibit complex flow patterns. The numerical tests include "lake at rest" steady state with very 
small perturbations as well as completely dry and almost dry areas. The obtained results confirm the capability of the scheme to preserve the positivity of $h$ and demonstrate that no instabilities are developed at the (almost) dry states. In the other numerical test, the results computed by the new scheme have been compared with those computed by the triangular central-upwind scheme. When the new scheme was used, the water surface component of the solution was resolved with higher resolution and was free of fluctuations. The velocity fields computed by the new scheme were also smooth while those computed by the triangular scheme were noisy.

We have also tested the proposed scheme on a problem with a transcritical flow over a bump. The computed water surface elevation and discharge were compared to the analytical ones. The results showed that the proposed central-upwind scheme is capable of quite accurately simulating such flows. In the next test, we have simulated a 2D dam break on a sloping flume with friction. The water surface values computed using a fully unstructured grids were compared to the experimental measurements and showed the ability of the developed scheme to take into account both complex domain geometry and a friction term. In the final numerical example, a dam break over a triangular obstacle was simulated and once again the obtained results were compared to the experimental measurements. This test helped us to confirm the ability of our quadrilateral central-upwind scheme to accurately predict the wetting and drying features of the flow. In summary, our numerical results confirmed robustness and high accuracy of the new scheme and also demonstrated its potential advantages over the triangular central-upwind scheme. 


\section{Acknowledgment}

This publication was partially made possible by NPRP Grant 4-935-2-354 from the Qatar

National Research Fund (a member of the Qatar Foundation) supporting the research of $A$.

Mohammadian \& H. Shirkhani. The work of A. Kurganov was supported in part by NSF grants

DMS-1216957 and DMS-1521009 and ONR grant N00014-12-1-0833.

\section{References}

1. Casulli V. Semi-implicit finite difference methods for the two-dimensional shallow water equations, Journal of Computational Physics 1990; 86(1): 56-74.

2. Xing YL, Shu CW. High order finite difference WENO schemes with the exact conservation property for the shallow water equations, Journal of Computational Physics 2005; 208 (1): 206-227.

3. Casulli V, Walters R.A. An unstructured grid, three-dimensional model based on the shallow water equations, Int. Jour. for Numerical Methods in Fluids 2000; 32: 331-348.

4. Xing Y, Shu C-W. A survey of high order schemes for the shallow water equations, J. Math. Study, 2014; 47: 221--249.

5. Hanert E, Le Roux DY, Legat V, Deleersnijder E. An efficient Eulerian finite element method for the shallow water equations. Ocean Modelling 2005; 10: 115-136.

6. White L, Deleersnijder E, Legat V. A three-dimensional unstructured mesh finite element shallow-water model, with application to the flows around an island and in a wind-driven, elongated basin. Ocean Modelling 2008; (22): 26-47.

7. Triki A. Multiple-grid finite element solution of the shallow water equations: Water hammer phenomenon, Computers \& Fluids 2014; 90:65-71.

8. Xing Y. Exactly well-balanced discontinuous galerkin methods for the shallow water equations with moving water equilibrium, J. Comput. Phys. 2014; 257: 536--553.

9. Xing Y, Zhang X, Shu C-W. Positivity-preserving high order well-balanced discontinuous Galerkin methods for the shallow water equations, Adv. Water Resour. 2010; 33 (12): 1476-1493.

10. Casulli V, Walters RA. An unstructured grid, three-dimensional model based on the shallow water equations, Int. J. Num. Methods in Fluids 2000; 32: 331-348.

11. Bonaventura L, Miglio E, Saleri F. Finite Volume Solvers for the Shallow Water Equations Using Matrix Radial Basis Function Reconstruction, Numerical Mathematics and Advanced Applications 2006; 207-214.

12. Mohamed K. A finite volume method for numerical simulation of shallow water models with porosity, Computers \& Fluids, 2014; (104):9-19. 
13. Castro MJ, Lopez-Garcia JA, Pares C. High order exactly well-balanced numerical methods for shallow water systems, J. Comput. Phys. 2013; 246: 242--264.

14. Gallardo J, Pares C, Castro M. On a well-balanced high-order finite volume scheme for shallow water equations with topography and dry areas, J. Comput. Phys. 2007; 227: 574--601.

15. Noelle S, Xing Y, Shu C-W. High-order well-balanced finite volume WENO schemes for shallow water equation with moving water, J. Comput. Phys. 2007; 226: 29--58.

16. Kurganov A, Tadmor E. New high resolution central schemes for nonlinear conservation laws and convection-diffusion equations. J. Comput. Phys. 2000; 160: 241-282.

17. Kurganov A, Noelle S, Petrova G. Semi-discrete central-upwind scheme for hyperbolic conservation laws and Hamilton-Jacobi equations. SIAM J. Sci. Comput. 2001; 23:707-740.

18. Kurganov A, Tadmor E. Solution of two-dimensional riemann problems for gas dynamics without riemann problem solvers. Numer. Methods Partial Differential Equations 2002; 18:584-608.

19. Kurganov A, Lin CT. On the reduction of numerical dissipation in central-upwind schemes. Commun. Comput. Phys. 2007; 2:141-163.

20. Kurganov A, Petrova G. Central-upwind schemes on triangular grids for hyperbolic systems of conservation laws. Numer. Methods Partial Diff. Equ. 2005; 21: 536-552.

21. Kurganov A, Levy D. Central-upwind schemes for the Saint-Venant system. ESAIM: M2AN 36 2002; $397-425$.

22. Kurganov A, Petrova G. A second-order well-balanced positivity preserving scheme for the Saint-Venant system. Commun. Math. Sci. 2007; 5:133-160.

23. Bryson S, Epshteyn Y, Kurganov A, Petrova G. Well-balanced positivity preserving central-upwind scheme on triangular grids for the Saint-Venant system. M2AN Math. Model. Numer. Anal. 2011; 45(3):423-446.

24. Beljadid A, Mohammadian A, Kurganov A. Well-Balanced Positivity Preserving Cell-Vertex CentralUpwind Scheme for Shallow Water Flow, (Submitted)

25. Chou SH, Kwak DY, Kim KY. Mixed finite volume methods on non-staggered quadrilateral grids for elliptic problems, Journal: Math. Comp. 2003; 72: 525-539.

26. Sheng Z, Yuan G. A Finite Volume Scheme For Diffusion Equations On Distorted Quadrilateral Meshes, Transport Theory And Statistical Physics, 2008; 37:171-207.

27. Feng X, Li R, He Y, Liu D. P1-Nonconforming Quadrilateral Finite Volume Methods for the Semilinear Elliptic Equations, J Sci Comput 2012; 52:519-545.

28. Xie B, Xiao F. Two and three dimensional multi-moment finite volume solver for incompressible NavierStokes equations on unstructured grids with arbitrary quadrilateral and hexahedral elements, Computers \& Fluids 2014; 104: 40-54. 
29. Kuirya SN, Senb D, Dinga Y. A high-resolution shallow water model using unstructured quadrilateral grids, Computers \& Fluids, 2012; 68: 16-28.

30. Wu WM, Sanchez A, Zhang ML. An implicit 2-D shallow water flow model on unstructured quadtree rectangular mesh. J Coastal Res, 2011; S1-59: 15-26.

31. Chou SH, Kwak DY, Vassilevski PS. Mixed upwinding covolume methods on rectangular grids for convection-diffusion problems, SIAM Journal on Scientific Computing 1999; 21 (1): 145-165.

32. Suli E. The accuracy of cell vertex finite volume methods on quadrilateral meshes, Math. Comp. 1992; 59: 359-382.

33. Kernkamp HWJ, Dam AV, Stelling GS, Goede, ED. Efficient scheme for the shallow water equations on unstructured grids with application to the Continental Shelf, Ocean Dynamics 2011; 61: 1175-1188.

34. Begnudelli L, Sanders BF. Conservative Wetting and Drying Methodology for Quadrilateral Grid FiniteVolume Models, J. Hydraul. Eng. 2007; 133: 312-322.

35. Liang Q, Du G, Hall JW, Borthwick AGL. Flood inundation modeling with an adaptive quadtree grid shallow water equation solver. J. Hydraulic Eng. 2008; 134(11): 1603-1610.

36. Greaves D. A quadtree adaptive method for simulating fluid flows with moving interfaces. J. Computational Physics 2004; 194(1): 35-56.

37. Alcrudo F, Garcia-Navarro P. A high-resolution Godunov-type scheme in finite volumes for the 2D shallow-water equations, Int. j. numer. methods fluids 1993; 16: 489-505.

38. Gottlieb S, Shu CW, Tadmor E. High order time discretization methods with the strong stability property. SIAM Rev. 2001; 43: 89-112.

39. Gottlieb S, Ketcheson D, Shu CW. Strong stability preserving Runge-Kutta and multistep time discretizations. World Scientific Publishing Co. Pte. Ltd., Hackensack, NJ, 2011.

40. LeVeque RJ. Balancing source terms and flux gradients in high-resolution Godunov methods: the quasisteady wave propagation algorithm. J. Comput. Phys. 1998; 146: 346-365.

41. Mohammadian A, Le Roux DY. Simulation of shallow flows over variable topographies using unstructured grids, Internat. J. Numer. Methods Fluids 2006; 52 (5): 473--498.

42. Goutal N, Maurel F. Dam-break wave simulation. Rep. HE43/97/016/B. Direction des études et recherches, Electricité de France, Paris, France, 1997.

43. Valiani A, Begnudelli, L. Divergence form for bed slope source term in shallow water equations, J. Hydraul. Eng. 2006; 132 (7): 652--665.

44. Hou, Jingming, et al. "A robust well-balanced model on unstructured grids for shallow water flows with wetting and drying over complex topography." Computer Methods in Applied Mechanics and Engineering 257 (2013): 126-149. 
45. Bellos CV, Soulis JV, Sakkas JG. Computation of two-dimensional dam-break-induced flows, Adv. Water Resour. 1991; 14 (1), 31- 41.

46. Liang Q, Marche F. Numerical resolution of well-balanced shallow water equations with complex source terms, Adv. Water Resour. 2009; 32 (6), 873--884.

47. Brufau P, Vázquez-Cendón ME, García-Navarro P. A numerical model for the flooding and drying of irregular domains, J. Numer. Method Fluid. 2002; 39: 247--275. 